\title{
LEVEL CROSSINGS OF ABSOLUTELY CONTINUOUS STATIONARY SYMMETRIC $\alpha$-STABLE PROCESSES
}

\author{
By ROBert AdleR ${ }^{1}$ AND Gennady SAMOROdnitsky ${ }^{2}$ \\ Technion-Israel Institute of Technology and Cornell University
}

We describe the mean rate at which a general absolutely continuous stationary $S \alpha S$ process crosses a high level. Only nondegeneracy assumptions are imposed in the case $1<\alpha<2$. The same results hold for $0<\alpha \leq 1$ under certain conditions, ensuring existence of the required conditional moments and the applicability of the classical integral formula for the expected number of level crossings.

1. Introduction. Let $\{X(t), t \in \Re\}$ be a real, stationary, symmetric, $\alpha$-stable $(S \alpha S)$ process, $0<\alpha<2$ with absolutely continuous sample paths, representable as

$$
X(t)=\int_{S} f(t, x) M(d x), \quad t \in \Re,
$$

where $(S, \mathscr{S})$ is a measurable space, $M$ is an independently scattered $\sigma$-additive $S \alpha S$ random measure on $(S, \mathscr{S})$ with a $\sigma$-finite control measure $m$, and $f(t, \cdot) \in L^{\alpha}(S, \mathscr{S}, m)$ for every $t \in \mathfrak{R}$. (cf. Samorodnitsky and Taqqu [19] for more details on $S \alpha S$ random measures and stochastic integrals with respect to them.)

For $\lambda \geq 0$, let $C_{\lambda}(T)$ be the number of upcrossings of the level $\lambda$ in the interval $[0, T]$, provided that this quantity is well defined. (cf. Cramér and Leadbetter [6] for a formal definition. We will deal with the issue of "well defined" below.) It is of fundamental importance for the application of stable processes to be able to say as much as possible about the distribution of $C_{\lambda}(T)$. In particular, one would like to be able to calculate the expectation $E\left(C_{\lambda}(T)\right)$. Of course, by stationarity and the continuity of $\alpha$-stable distributions, $E\left(C_{\lambda}(T)\right)=T E\left(C_{\lambda}(1)\right)$, so that the parameter $T$ is not important. We will study, therefore, $E C_{\lambda}=E\left(C_{\lambda}(1)\right)$.

\footnotetext{
Received January 1996; revised November 1996.

${ }^{1}$ Research supported by United States-Israel Binational Science Foundation Grant 92-00074 and by ONR Grant N00014-94-1-0191.

${ }^{2}$ Research supported by United States-Israel Binational Science Foundation Grant 92-00074, NSF Grant DMS-94-00535 and NSA Grant MDA904-95-H-1036. Part of Samorodnitsky's research was done at ETH, Zürich, under support of Forschungsinstitut für Mathematik and Institut für Operations Research; another part was done at Chalmers University, Göteborg, under support of Swedish Natural Science Research Council Grant F-GF 09207-305.

AMS 1991 subject classifications. Primary 60G10, 60G70; secondary 60G17, 60G18.

Key words and phrases. Stable processes, level crossings.
} 
In the well-known Gaussian case, the famous Rice formula

$$
E C_{\lambda}=\frac{1}{2 \pi}\left(\frac{-R^{\prime \prime}(0)}{R(0)}\right)^{1 / 2} \exp \left(-\frac{\lambda^{2}}{2 R(0)}\right)
$$

gives the expected number of level upcrossings for a stationary Gaussian process with a covariance function $R$. See [16] and [6].

The Rice formula is generally derived in two stages. In the first, $E C_{\lambda}$ is represented as a general expression involving the joint (bivariate normal) density of the process and its derivative at a given point. This expression is then evaluated via a straightforward exercise in integration. We shall show below that, under appropriate conditions, the first stage of this argument also carries over to the stable situation. However, since even the univariate density of a stable random variable is generally not available in closed form, the second stage of this argument cannot be carried out, and so a precise, closed form expression for $E C_{\lambda}$ is not accessible in the general stable case $0<\alpha<2$. The route that one is forced to take is therefore one of bounds and asymptotics.

The first result of this type is due to Marcus [12], and deals with a particular class of stationary $S \alpha S$ processes, that of the real, harmonizable ones. Adopting the representation (1.1), these have $S=R \times \Omega^{\prime}$ and $\mathscr{S}=\mathscr{B} \times$ $\mathscr{F}^{\prime}$, where $\left(\Omega^{\prime}, \mathscr{F}^{\prime}, P^{\prime}\right)$ is a probability space supporting two independent standard normal random variables $G_{1}$ and $G_{2}$. Furthermore, $m=F \times P^{\prime}(F$ being a finite measure on $\mathfrak{R}$ ), and

$$
f\left(t,\left(y, \omega^{\prime}\right)\right)=G_{1}\left(\omega^{\prime}\right) \cos t y+G_{2}\left(\omega^{\prime}\right) \sin t y, \quad y \in \Re, \omega^{\prime} \in \Omega^{\prime} .
$$

An alternative, and probably more familiar, representation in this case is

$$
X(t)=a_{\alpha} \operatorname{Re}\left\{\int_{-\infty}^{\infty} e^{i t x} \tilde{M}(d x)\right\}, \quad t \in \Re,
$$

where $\tilde{M}$ is a complex valued rotationally invariant $S \alpha S$ random measure with the same control measure $m$, and $a_{\alpha}$ is a constant depending only on $\alpha$.

Using the fact that a real harmonizable stationary $S \alpha S$ process can be written as a mixture of stationary Gaussian processes, Marcus [12] applied the Rice formula conditionally, allowing him to derive bounds on the expected number of level upcrossings. Later, Adler, Samorodnitsky and Gadrich [1] improved Marcus' results for harmonizable processes, while using the same approach. In particular, they showed that, if the process is regular enough to guarantee the finiteness of $E C_{0}$, then

$$
\lim _{\lambda \rightarrow \infty} \lambda^{\alpha} E C_{\lambda}=\kappa_{\alpha} \lambda_{1}
$$

where for $0<\alpha<2, \kappa_{\alpha}$ is a finite positive constant depending only on $\alpha$, and

$$
\lambda_{1}=\int_{-\infty}^{\infty}|x| F(d x) .
$$


Given the success of this approach in the harmonizable case, it is natural to try to extend it to more general stable processes, particularly in view of the fact that essentially all the $S \alpha S$ processes described above can, in fact, be represented as mixtures of Gaussians. However, in the general case, the conditional Gaussian processes are no longer stationary, so that the simple Rice formula no longer applies to them and the computations involved become forbiddingly complicated. Consequently, there has been no further development along this line.

In the present paper we shall determine the asymptotic behavior of the expected number of level upcrossings for general, stationary, $S \alpha S$ processes, obtaining a result similar to (1.2). Clearly, we need to take a different path from that described above, based on a specific, mixed Gaussian, representation of $S \alpha S$ processes. While more general representations are also available and the overall structure of stationary $S \alpha S$ processes is understood today much better than a few years ago (primarily due to the work of J. Rosinski; cf. [18]), our approach will not use this general structure either. Instead, we will, essentially, revert to first principles, and proceed as follows.

Let $\{X(t), t \in \mathfrak{R}\}$ be a stationary $S \alpha S$ process given in the form (1.1). We will assume that for $m$ almost every $x \in S, f(\cdot, x)$ is an absolutely continuous function, with

$$
f(t, x)=f(0, x)+\int_{0}^{t} \dot{f}(s, x) d s, \quad 0 \leq t \leq 1
$$

such that $\dot{f}:[0,1] \times S \rightarrow \Re$ is jointly measurable and satisfies one of the following three conditions:

$$
\begin{gathered}
\int_{0}^{1}\left(\int_{S}|\dot{f}(t, x)|^{\alpha} m(d x)\right)^{1 / \alpha} d t<\infty \quad \text { if } 1<\alpha<2, \\
\int_{0}^{1} \int_{S}|\dot{f}(t, x)|\left[1+\log _{+} \frac{|\dot{f}(t, x)| \int_{0}^{1} \int_{S}|\dot{f}(u, v)| d u m(d v)}{\int_{S}|\dot{f}(t, v)| m(d v) \int_{0}^{1}|\dot{f}(u, x)| d u}\right] m(d x) d t \\
<\infty \quad \text { if } \alpha=1,
\end{gathered}
$$

where, for $a>0, \log _{+} a=\max (\log a, 0)$, and

$$
\int_{S}\left(\int_{0}^{1}|\dot{f}(t, x)| d t\right)^{\alpha} m(d x)<\infty \quad \text { if } 0<\alpha<1 .
$$

Under these conditions, it follows from [17] that $\{X(t), t \in \Re\}$ has an absolutely continuous version (which we henceforth identify with the process itself), and that is derivative in the sense of absolute continuity is given by

$$
\dot{X}(t)=\int_{S} \dot{f}(t, x) M(d x), \quad 0 \leq t \leq 1 .
$$

(See also Section 11.7 of [19].) 
(These conditions are not only sufficient for existence of an absolutely continuous version of the process, but also necessary for it. Furthermore, they are not as forbidding or as difficult to check as they may seem. Example 1.1 below shows how they simplify in the case of a stationary moving average process.)

The first step towards developing an asymptotic formula for $C_{\lambda}$ will be to establish when we are justified in using the standard, exact, formula

$$
E C_{\lambda}=\int_{0}^{\infty} y g(\lambda, y) d y,
$$

where $g$ is the joint density of $X(0)$ and $\dot{X}(0)$ (see, e.g., Theorem 7.2.4 of [9]). We will see that, apart from nondegeneracy conditions, in the case $1<\alpha<2$, the integral formula (1.7) holds for any absolutely continuous $S \alpha S$ process as above. In the case $0<\alpha \leq 1$ we will have to impose certain additional regularity assumptions on the process $\{X(t), t \in \mathfrak{R}\}$ to justify (1.7).

As mentioned above, it would be nice to be able to actually evaluate (1.7), but this is not possible. Hence we turn to a study of the asymptotic behavior of the integral in the right-hand side of (1.7) as $\lambda \rightarrow \infty$. The two steps in the derivation are mathematically independent of one another and appear in the Sections 3 and 4, respectively.

Our approach to the asymptotic behavior of the integral is, once again, via conditional Gaussianity. However, instead of dealing with the entire process, we merely rely on the facts that an $S \alpha S$ process and its derivative at any fixed time are jointly $S \alpha S$, and that any $S \alpha S$ random vector is a mixture of Gaussian random vectors. Thus the joint density $g$ in (1.7) can be viewed as a mixture of bivariate normal densities, and the corresponding integral for a bivariate normal density can, to a certain extent, be simplified. The problem is then to determine the asymptotic behavior of the expectation of the resulting expression, and the reader will find the details of that in Section 4 .

This approach leads to the following theorem, which is the main result of this paper.

THEOREM 1.1. Let $\{X(t), t \in \Re\}$ be a real stationary $S \alpha S$ process given in the form (1.1) satisfying (1.3), (1.4) or (1.5) (depending on the value of $\alpha$ ), with absolutely continuous sample paths satisfying (1.6). Assume that for every $\gamma_{i} \in \mathfrak{R}, i=1,2, \gamma_{1}+\gamma_{2} \neq 0$,

$$
m\left\{x \in S: \gamma_{1} f(0, x)-\gamma_{2} \dot{f}(0, x) \neq 0\right\}>0,
$$

and for all $n$ large enough and $\gamma_{i} \in \mathfrak{R}, i=1,2$,

$$
m\left\{x \in S: \gamma_{1} f\left(2^{-n}, x\right)-\gamma_{2} f(0, x) \neq 0\right\}>0 .
$$

If $\alpha=1$, we assume additionally that

$$
\int_{S}|\dot{f}(0, x) \log | \frac{\dot{f}(0, x)}{f(0, x)}|| m(d x)<\infty .
$$


If $0<\alpha<1$, we assume additionally that

$$
\int_{S} \frac{|\dot{f}(0, x)|}{|f(0, x)|^{1-\alpha}} m(d x)<\infty .
$$

Finally, in the case $0<\alpha \leq 1$ we assume that there is $a \delta>0$ and $0<M<\infty$ such that for all $n$ large enough and $t \in\left(0,2^{-n}\right]$,

$$
\int_{S} \frac{\left|2^{n}\left(f\left(2^{-n}, x\right)-f(0, x)\right)\right|^{1+\delta}}{\left|2^{n} t\left(f\left(2^{-n}, x\right)-f(0, x)\right)+f(0, x)\right|^{1+\delta-\alpha}} m(d x) \leq M .
$$

Then (1.7) holds for every $\lambda \in \Re$, and, furthermore,

$\lim _{\lambda \rightarrow \infty} \lambda^{\alpha} E C_{\lambda}$

$$
\begin{aligned}
= & \alpha 2^{\alpha-1 / 2} \pi^{-(1+1 / \alpha) / 2}\left(\Gamma\left(\frac{\alpha+1}{2}\right)\right)^{1+1 / \alpha} C_{\alpha}^{-1 / \alpha} \\
& \times \int_{S}|\dot{f}(0, x)||f(0, x)|^{\alpha-1} \mathbf{1}(f(0, x) \dot{f}(0, x)>0) m(d x),
\end{aligned}
$$

where $C_{\alpha}$ is given by (2.11).

The following is an immediate application of this result.

EXAMPLE 1.1. An important class of stationary $S \alpha S$ processes is that of moving averages. These are processes of the form

$$
X(t)=\int_{-\infty}^{\infty} f(t+x) M(d x), \quad t \in \Re,
$$

where the $S \alpha S$ random measure $M$ has Lebesgue control measure on $\Re$, and $f \in L^{\alpha}(R)$. Unlike the harmonizable stationary processes described above, moving average processes are mixing [13], and so provide an attractive modeling tool. To ensure absolute continuity of a moving average process, we need to assume (cf. [19], Section 11.7) that

$$
f(b)-f(a)=\int_{a}^{b} \dot{f}(s) d s, \quad a<b,
$$

with $\dot{f}$ satisfying

$$
\int_{-\infty}^{\infty}|\dot{f}(x)|^{\alpha} d x<\infty \quad \text { if } 1<\alpha<2,
$$

$$
\begin{aligned}
\int_{0}^{1} \int_{-\infty}^{\infty}|\dot{f}(x)|\left[1+\log _{+} \frac{|\dot{f}(x)|}{F_{1}(x+t)}\right] d x d t<\infty \quad \text { if } \alpha=1, \\
\int_{-\infty}^{\infty} F_{1}(x)^{\alpha} d x<\infty \quad \text { if } 0<\alpha<1 .
\end{aligned}
$$


Here

$$
F_{1}(x)=\int_{x}^{x+1}|\dot{f}(s)| d s .
$$

It is obvious that, in this case, conditions (1.8) and (1.9) hold automatically as long as the process is not identically equal to 0 . The conditions (1.10), (1.11) and (1.12) take, in the present case, the form of, correspondingly,

$$
\begin{gathered}
\int_{-\infty}^{\infty}|\dot{f}(x) \log | \frac{\dot{f}(x)}{f(x)}|| d x<\infty, \\
\int_{-\infty}^{\infty} \frac{|\dot{f}(x)|}{|f(x)|^{1-\alpha}} d x<\infty
\end{gathered}
$$

and

$$
\int_{-\infty}^{\infty} \frac{\left|2^{n}\left(f\left(2^{-n}+x\right)-f(x)\right)\right|^{1+\delta}}{\left|2^{n} t\left(f\left(2^{-n}+x\right)-f(x)\right)+f(x)\right|^{1+\delta-\alpha}} d x \leq M .
$$

Therefore, for every absolutely continuous stationary $S \alpha S$ moving average process with $1<\alpha<2$ [or with $0<\alpha \leq 1$, under (1.16), (1.17) and (1.18)] we have

$$
\begin{aligned}
\lim _{\lambda \rightarrow \infty} \lambda^{\alpha} E C_{\lambda}= & \alpha 2^{\alpha-1 / 2} \pi^{-(1+1 / \alpha) / 2}\left(\Gamma\left(\frac{\alpha+1}{2}\right)\right)^{1+1 / \alpha} C_{\alpha}^{-1 / \alpha} \\
& \times \int_{-\infty}^{\infty}|\dot{f}(x)||f(x)|^{\alpha-1} \mathbf{1}(f(x) \dot{f}(x)>0) d x,
\end{aligned}
$$

with $C_{\alpha}$ given by (2.11). To give an even more explicit example, let $\{X(t)$, $t \in \mathfrak{R}\}$ be the two-sided Ornstein-Uhlenbeck process, that is, a moving average process with

$$
f(x)=(\alpha \gamma / 2)^{1 / \alpha} e^{-\gamma|x|}, \quad x \in \Re, \gamma>0 .
$$

Then the conditions (1.10), (1.11) and (1.12) are easily seen to hold, and so we have

(1.20) $\lim _{\lambda \rightarrow \infty} \lambda^{\alpha} E C_{\lambda}=\alpha 2^{\alpha-1 / 2} \pi^{-(1+1 / \alpha) / 2}\left(\Gamma\left(\frac{\alpha+1}{2}\right)\right)^{1+1 / \alpha} C_{\alpha}^{-1 / \alpha} \gamma^{\alpha-1}$

for all $0<\alpha<2$ and $\gamma>0$.

We now turn to the proof of Theorem 1.1. The following section contains certain technical lemmas needed later. As noted above, the main work is in Sections 3 and 4 .

2. Some lemmas. We collect in this section some technical results that will be used later.

LEMMA 2.1. Let $R_{1}, R_{2}, \ldots$ be a sequence of i.i.d. nonnegative random variables independent of the sequence $\Gamma_{1}, \Gamma_{2}, \ldots$ of the arrival times of a unit rate Poisson process. Let $0<\alpha<1$. Assume that $E R_{1}^{\alpha}<\infty$. 
(i) The sum $\sum_{j=1}^{\infty} \Gamma_{j}^{-1 / \alpha} R_{j}$ converges with probability 1 , and

$$
\lim _{\lambda \rightarrow \infty} \lambda^{\alpha} P\left(\sum_{j=2}^{\infty} \Gamma_{j}^{-1 / \alpha} R_{j}>\lambda\right)=0 .
$$

Moreover, if $E R_{1}<\infty$, then for every $\lambda>0$,

$$
P\left(\sum_{j=2}^{\infty} \Gamma_{j}^{-1 / \alpha} R_{j}>\lambda\right) \leq C \lambda^{-\min (1,2 \alpha)}
$$

for some finite positive constant $C$ depending on $\alpha$ and the distribution of $R_{1}$.

(ii) Assume that

$$
E\left(R_{1}^{\alpha} \log \left(1+R_{1}\right)\right)<\infty .
$$

Then

$$
E\left(\sum_{j=1}^{\infty} j^{-1 / \alpha} R_{j}\right)^{\alpha}<\infty
$$

(iii) Let

$$
F=\frac{\sum_{j=1}^{\infty} \Gamma_{j}^{-1 / \alpha} R_{j}}{\sum_{j=1}^{\infty} \Gamma_{j}^{-1 / \alpha}} .
$$

Then for any $p>\alpha, E F^{p}<\infty$ if and only if $E R_{1}^{p}<\infty$. Moreover,

$$
c_{\alpha, p}^{-1} E R_{1}^{p} \leq E F^{p} \leq c_{\alpha, p} E R_{1}^{p}
$$

for some $c_{\alpha, p} \in(0, \infty)$ that depends only on $\alpha$ and $p$.

Furthermore, under the assumption of part (ii) we have $E F^{\alpha}<\infty$, and, moreover,

$$
E F^{\alpha} \leq c_{\alpha}\left(E\left(R_{1}^{\alpha} \log \left(2+R_{1}\right)\right)+E R_{1}^{\alpha} \log \frac{1}{E R_{1}^{\alpha}}\right)
$$

for some $c_{\alpha} \in(0, \infty)$ that depends only on $\alpha$.

Proof. (i) The fact that the series converges with probability 1 is well known (e.g., Theorem 1.4.5 in [19]), and one shows (2.1) by retracing the steps of the proof of Property 1.4.4 there. For (2.2) with $1 / 2<\alpha<1$, note that $E\left(\sum_{j=2}^{\infty} \Gamma_{j}^{-1 / \alpha} R_{j}\right)<\infty$, while in the case $0<\alpha \leq 1 / 2$, note that for all $j \geq 2$ we have $P\left(\Gamma_{j}^{-1 / \alpha} R_{j}>\lambda\right) \leq C \lambda^{-2 \alpha}$, while $E\left(\sum_{j=K}^{\infty} \Gamma_{j}^{-1 / \alpha} R_{j}\right)<\infty$ for $K$ large enough. Hence (2.2) follows in this case, since for every $K$ we have

$$
\begin{aligned}
& P\left(\sum_{j=2}^{\infty} \Gamma_{j}^{-1 / \alpha} R_{j}>\lambda\right) \\
& \quad \leq \sum_{j=2}^{K} P\left(\Gamma_{j}^{-1 / \alpha} R_{j}>\lambda / 2 K\right)+P\left(\sum_{j=K+1}^{\infty} \Gamma_{j}^{-1 / \alpha} R_{j}>\lambda / 2\right) .
\end{aligned}
$$


(ii) This is a consequence of Proposition 5.2 of [3].

(iii) Clearly,

$$
E F^{p} \geq E R_{1}^{p} E\left(\frac{\Gamma_{1}^{-1 / \alpha}}{\sum_{j=1}^{\infty} \Gamma_{j}^{-1 / \alpha}}\right)^{p},
$$

proving the left-hand side inequality in (2.4). To prove the other inequality we consider two cases.

Suppose first that $p \geq 1$. Then by Hölder's inequality,

$$
F^{p}=\left(\sum_{j=1}^{\infty} \frac{\Gamma_{j}^{-1 / \alpha}}{\sum_{i=1}^{\infty} \Gamma_{i}^{-1 / \alpha}} R_{j}\right)^{p} \leq \sum_{j=1}^{\infty} \frac{\Gamma_{j}^{-1 / \alpha}}{\sum_{i=1}^{\infty} \Gamma_{i}^{-1 / \alpha}} R_{j}^{p},
$$

and so $E F^{p} \leq E R_{1}^{p}$. If $\alpha<p<1$, set $K=[2 p / \alpha]$ and note that for every $k>K, E \Gamma_{k}^{-2 p / \alpha}<\infty$, which implies that

$$
E\left(\sum_{j=K+1}^{\infty} \Gamma_{j}^{-p / \alpha}\right)^{2}<\infty .
$$

Therefore,

$$
\begin{aligned}
E F^{p} & \leq E\left(\sum_{j=1}^{K} R_{j}^{p}+\frac{\sum_{j=K+1}^{\infty} \Gamma_{j}^{-p / \alpha} R_{j}^{p}}{\left(\sum_{j=1}^{\infty} \Gamma_{j}^{-1 / \alpha}\right)^{p}}\right) \\
& \leq E R_{1}^{p}\left(K+E \frac{\sum_{j=K+1}^{\infty} \Gamma_{j}^{-p / \alpha}}{\left(\sum_{j=1}^{\infty} \Gamma_{j}^{-1 / \alpha}\right)^{p}}\right) \\
& \leq E R_{1}^{p}\left(K+\left(E\left(\sum_{j=K+1}^{\infty} \Gamma_{j}^{-p / \alpha}\right)^{2}\left(E\left(\sum_{j=1}^{\infty} \Gamma_{j}^{-1 / \alpha}\right)^{-2 p}\right)^{1 / 2}\right),\right.
\end{aligned}
$$

thus proving the second inequality in (2.4) in all cases.

For (2.5) let

$$
a_{n}=n^{(1-\alpha) / 2 \alpha}\left(E R_{1}^{\alpha}\right)^{1 / \alpha}, \quad n \geq 1 .
$$

We have

$$
\begin{aligned}
E F^{\alpha} \leq & E\left(\frac{\sum_{j=1}^{\infty} \Gamma_{j}^{-1 / \alpha} R_{j} \mathbf{1}\left(R_{j} \leq a_{j}\right)}{\sum_{j=1}^{\infty} \Gamma_{j}^{-1 / \alpha}}\right)^{\alpha} \\
& +E\left(\frac{\sum_{j=1}^{\infty} \Gamma_{j}^{-1 / \alpha} R_{j} \mathbf{1}\left(R_{j}>a_{j}\right)}{\sum_{j=1}^{\infty} \Gamma_{j}^{-1 / \alpha}}\right)^{\alpha}:=E F_{1}^{\alpha}+E F_{2}^{\alpha} .
\end{aligned}
$$


Clearly, taking now $K=\left[1 / \alpha^{1 / 2}\right]+1$, one has

$$
\begin{aligned}
E F_{1}^{\alpha} \leq & E\left(\frac{\sum_{j=1}^{\infty} \Gamma_{j}^{-1 / \alpha} a_{j}}{\sum_{j=1}^{\infty} \Gamma_{j}^{-1 / \alpha}}\right)^{\alpha} \leq E R_{1}^{\alpha} E\left(\frac{\sum_{j=1}^{\infty} \Gamma_{j}^{-1 / \alpha} j^{(1-\alpha) / 2 \alpha}}{\sum_{j=1}^{\infty} \Gamma_{j}^{-1 / \alpha}}\right)^{\alpha} \\
\leq & E R_{1}^{\alpha}\left[K^{(1-\alpha) / 2 \alpha}+E\left(\frac{\sum_{j=K+1}^{\infty} \Gamma_{j}^{-1 / \alpha} j^{(1-\alpha) / 2 \alpha}}{\sum_{j=1}^{\infty} \Gamma_{j}^{-1 / \alpha}}\right)^{\alpha}\right] \\
\leq & E R_{1}^{\alpha}\left[K^{(1-\alpha) / 2 \alpha}+\left(E\left(\sum_{j=K+1}^{\infty} \Gamma_{j}^{-1 / \alpha} j^{(1-\alpha) / 2 \alpha}\right)^{\alpha^{1 / 2}}\right)^{\alpha^{1 / 2}}\right. \\
& \left.\times\left(E\left(\sum_{j=1}^{\infty} \Gamma_{j}^{-1 / \alpha}\right)^{-\alpha /\left(1-\alpha^{1 / 2}\right)}\right)^{1-\alpha^{1 / 2}}\right] \\
& =c_{\alpha} E R_{1}^{\alpha},
\end{aligned}
$$

where $c_{\alpha}$ is a finite positive constant that depends only on $\alpha$ and that may change from line to line. To see why the latter two expectations are finite, one only has to recall the two simple facts that for any $p \in \Re$ we have $E \Gamma_{j}^{p} \sim z_{p} j^{p}$ as $p \rightarrow \infty$, where $z_{p}$ is a finite positive constant that depends only on $p$, and that a positive $\alpha$-stable random variable $(0<\alpha<1)$ has a density that decays faster than exponentially fast at the origin, so that it has negative moments of all orders. These two observations will be used throughout this paper, often without additional comment.

It is easy to check (using, for example, the fact that the event $\Gamma_{j}<j / 2$ has low probability) that

$$
E\left(\sup _{j>2} \frac{j}{\Gamma_{j}}\right)^{2}<\infty .
$$

Then, as before, we have

$$
\begin{aligned}
E F_{2}^{\alpha} & \leq 2 E R_{1}^{\alpha}+E \frac{\sum_{j=3}^{\infty} \Gamma_{j}^{-1} R_{j}^{\alpha} \mathbf{1}\left(R_{j}>a_{j}\right)}{\left(\sum_{j=1}^{\infty} \Gamma_{j}^{-1 / \alpha}\right)^{\alpha}} \\
& \leq 2 E R_{1}^{\alpha}+E \frac{\sup _{j>2} j / \Gamma_{j}}{\left(\sum_{j=1}^{\infty} \Gamma_{j}^{-1 / \alpha}\right)^{\alpha}} \sum_{j=3}^{\infty} j^{-1} E R_{1}^{\alpha} \mathbf{1}\left(R_{1}>a_{j}\right) \\
& \leq c_{\alpha} E\left[R_{1}^{\alpha}\left[1+\sum_{j=1}^{\infty} j^{-1} \mathbf{1}\left(j \leq\left(R_{1} /\left(E R_{1}^{\alpha}\right)^{1 / \alpha}\right)^{2 \alpha /(1-\alpha)}\right)\right]\right] \\
& \leq c_{\alpha}\left(E\left(R_{1}^{\alpha} \log \left(2+R_{1}\right)\right)+E R_{1}^{\alpha} \log \frac{1}{E R_{1}^{\alpha}}\right) .
\end{aligned}
$$

Now (2.5) follows from (2.6), (2.7) and (2.9). This completes the proof of the lemma. 
We will also need the following lemma.

LeMma 2.2. (i) Let $X$ be a positive, $\alpha / 2$ strictly stable random variable with scale parameter $\sigma$, so that it has Laplace transform $E \exp \{-\theta X\}=$ $\exp \left(-\sigma^{\alpha / 2} \theta^{\alpha / 2} / \cos (\pi \alpha / 4)\right)$. Then for any $p>-\alpha / 2$ we have

$$
\lim _{\lambda \rightarrow \infty} \lambda^{\alpha+2 p} E\left(X^{-p} \exp \left(-\lambda^{2} / X\right)\right)=\sigma^{\alpha / 2}(\alpha / 2) C_{\alpha / 2} \Gamma(p+\alpha / 2),
$$

where for $0<\alpha<2, C_{\alpha}$ is given by

$$
C_{\alpha}=\left(\int_{0}^{\infty} x^{-\alpha} \sin x d x\right)^{-1} .
$$

(ii) Let $0<\alpha<2$ and let $X$ be a nonnegative random variable such that $P(X>\lambda)=o\left(\lambda^{-\alpha / 2}\right)$ as $\lambda \rightarrow \infty$. Then for every $p>-\alpha / 2$,

$$
\lim _{\lambda \rightarrow \infty} \lambda^{\alpha+2 p} E\left(X^{-p} \exp \left(-\lambda^{2} / X\right)\right)=0 .
$$

Proof. (i) Let $f$ denote the density function of $X$. Then

$$
f(x) \sim(\alpha / 2) C_{\alpha / 2} \sigma^{\alpha / 2} x^{-(1+\alpha / 2)} \text { as } x \rightarrow \infty .
$$

(See, e.g., Property 1.2.15 in [19] and use the eventual monotonicity of the density.) Therefore, for any fixed $M>0$,

$$
\begin{aligned}
\lim _{\lambda \rightarrow \infty} \lambda^{\alpha+2 p} E\left(X^{-p} \exp \left(-\lambda^{2} / X\right)\right) \\
\quad=(\alpha / 2) C_{\alpha / 2} \sigma^{\alpha / 2} \lim _{\lambda \rightarrow \infty} \lambda^{\alpha+2 p} \int_{M}^{\infty} x^{-p} \exp \left(-\lambda^{2} / x\right) x^{-(1+\alpha / 2)} d x \\
=(\alpha / 2) C_{\alpha / 2} \sigma^{\alpha / 2} \int_{0}^{\infty} \exp (-1 / x) x^{-(p+1+\alpha / 2)} d x \\
=(\alpha / 2) C_{\alpha / 2} \sigma^{\alpha / 2} \Gamma(p+\alpha / 2),
\end{aligned}
$$

thus establishing (2.10).

(ii) Suppose first that $-\alpha / 2<p \leq 0$. Observe that for every $\varepsilon>0$ there is an $a \geq 0$ such that $X \leq_{\mathrm{st}} \varepsilon Y_{\alpha / 2}+a$, where $Y_{\alpha / 2}$ is a positive, $\alpha / 2$ strictly stable random variable with scale parameter $\sigma=1$. Therefore, letting $C$ be a finite positive constant that may change from line to line, we have

$$
\begin{aligned}
& E\left(X^{-p} \exp \left(-\lambda^{2} / X\right)\right) \\
& \leq E\left(\left(\varepsilon Y_{\alpha / 2}+a\right)^{-p} \exp \left(-\lambda^{2} /\left(\varepsilon Y_{\alpha / 2}+a\right)\right)\right) \\
&= E\left(\left(\varepsilon Y_{\alpha / 2}+a\right)^{-p} \exp \left(-\lambda^{2} /\left(\varepsilon Y_{\alpha / 2}+a\right)\right) \mathbf{1}\left(\varepsilon Y_{\alpha / 2} \leq a\right)\right) \\
&+E\left(\left(\varepsilon Y_{\alpha / 2}+a\right)^{-p} \exp \left(-\lambda^{2} /\left(\varepsilon Y_{\alpha / 2}+a\right)\right) \mathbf{1}\left(\varepsilon Y_{\alpha / 2}>a\right)\right) \\
& \leq C \exp \left(-\lambda^{2} / C\right)+E\left(\left(2 \varepsilon Y_{\alpha / 2}\right)^{-p} \exp \left(-\lambda^{2} /\left(2 \varepsilon Y_{\alpha / 2}\right)\right)\right) .
\end{aligned}
$$


We conclude by part (ii) of this lemma that

$$
\limsup _{\lambda \rightarrow \infty} \lambda^{\alpha+2 p} E\left(X^{-p} \exp \left(-\lambda^{2} / X\right)\right) \leq C \varepsilon^{\alpha / 2},
$$

and since $\varepsilon$ can be taken arbitrarily close to zero, our claim follows. If $p>0$, use the elementary observation that for any such $p$ and $\lambda>0$,

$$
\sup _{z>0} z^{-p} \exp \left(-\lambda^{2} / z\right)=c_{p} \lambda^{-2 p}
$$

for a $c_{p} \in(0, \infty)$, to conclude that

$$
E\left(X^{-p} \exp \left(-\lambda^{2} / X\right)\right) \leq C \lambda^{-2 p} E\left(\exp \left(-\lambda^{2} / 2 X\right)\right),
$$

and apply the case $p=0$, which we have just established.

3. The expected number of level upcrossings. This section contains the proof of the main result of the paper, Theorem 1.1. As we mentioned above, this amounts to justifying the formula (1.7). The asymptotic behavior of the integral appearing in the right-hand side of this formula is computed in the next section.

To justify the integral formula (1.7) we start with the approach of Marcus [11] to level crossings. Other available results, such as those of Brillinger [2] or Geman and Horowitz [7] only deliver (1.7) for almost every, but not for every, $\lambda$. The result of Michna and Rychlik [14] also establishes (1.7) for $S \alpha S$ processes. However, it assumes continuous differentiability for the process, for which tight conditions are unknown (see, e.g., Chapter 12 of [10], Chapter 10 of [19] and [15]). For completeness, we list below the conditions of Theorem 2.1 of Marcus [11] (simplified to our particular case, of a stationary process and the first moment only) that we will check to establish the validity of (1.7).

Let $\{X(t), t \in \Re\}$ be a stationary stochastic process with absolutely continuous sample paths. Let $g(x, y)$ denote the joint density function of $(X(0), \dot{X}(0))$, with $\dot{X}$ being the derivative in the sense of absolute continuity. Let

$$
h(\lambda)=\int_{0}^{\infty} y g(\lambda, y) d y .
$$

Furthermore, for $n \geq 1$ and $t \in\left[0,2^{-n}\right)$, let $g_{t, n}(x, y)$ denote the joint density of $2^{n} t\left(X\left(2^{-n}\right)-X(0)\right)+X(0)$ and $2^{n}\left(X\left(2^{-n}\right)-X(0)\right)$, and set

$$
h_{t, n}(\lambda)=\int_{0}^{\infty} y g_{t, n}(\lambda, y) d y \text {. }
$$

Then Marcus (Theorem 2.1, Marcus [11]) sets out a set of seven conditions that guarantee the veracity of (1.7). These are the following.

(M1) The density $g$ exists.

(M2) The function $h$ is bounded in a neighborhood of $\lambda$.

(M3) The function $h$ is continuous at $\lambda$.

(M4) The density $g_{t, n}$ exists for all $n$ large enough and $t \in\left[0,2^{-n}\right)$. 
(M5) The functions $h_{t, n}$ are uniformly bounded in a neighborhood of $\lambda$, for all $n$ large enough and all $t \in\left[0,2^{-n}\right)$.

(M6) The function $h_{t, n}$ is continuous at $\lambda$ for all $n$ large enough and all $t \in\left[0,2^{-n}\right)$.

(M7) For any sequence $\left\{t_{n}\right\}$ such that $t_{n} \in\left[0,2^{-n}\right)$ for all $n$ we have $\lim _{n \rightarrow \infty} h_{t_{n}, n}(\lambda)=h(\lambda)$.

With these conditions in front of us, we can now commence the proof of our main result.

Proof of Theorem 1.1. As a first step, we need to check the veracity of (1.7), so that all we really need do is to check that the conditions of our theorem guarantee that Marcus' (M1)-(M7) are satisfied.

To simplify things, assume for the moment that the control measure $m$ of the $S \alpha S$ random measure $M$ is a probability measure on $S$.

It follows from (1.8) that the $S \alpha S$ random vector $(X(0), \dot{X}(0))$ is not concentrated on any proper subspace of $\Re^{2}$, and so it has an absolutely continuous distribution with respect to the Lebesgue measure there. This verifies condition (M1) above.

To check the conditions (M2) and (M3) we observe that, since $m$ is a probability measure, we can write (in distribution)

$$
\begin{aligned}
& X(0)=b_{\alpha} \sum_{j=1}^{\infty} G_{j} \Gamma_{j}^{-1 / \alpha} f\left(0, U_{j}\right), \\
& \dot{X}(0)=b_{\alpha} \sum_{j=1}^{\infty} G_{j} \Gamma_{j}^{-1 / \alpha} \dot{f}\left(0, U_{j}\right),
\end{aligned}
$$

where

$$
b_{\alpha}=\left(2^{-\alpha / 2} \pi^{1 / 2}\left(\Gamma\left(\frac{\alpha+1}{2}\right)\right)^{-1} C_{\alpha}\right)^{1 / \alpha},
$$

and where $G_{j}, j \geq 1, \Gamma_{j}, j \geq 1$, and $U_{j}, j \geq 1$, are three independent sequences of random variables, such that $G_{j}, j \geq 1$, are i.i.d. standard normal random variables, $\Gamma_{j}, j \geq 1$, are the arrival times of a unit rate Poisson process on $(0, \infty)$, and $U_{j}, j \geq 1$, are i.i.d. $S$-valued random variables with a common law $m$. See, for example, Chapter 3 of [19]. Observe that, conditionally on $\Gamma_{j}, j \geq 1$, and $U_{j}, j \geq 1$, the random variables $X(0)$ and $\dot{X}(0)$ are jointly normal, with zero means and a variance-covariance matrix with elements

$$
\begin{aligned}
& \sigma_{1}^{2}=b_{\alpha}^{2} \sum_{j=1}^{\infty} \Gamma_{j}^{-2 / \alpha} f\left(0, U_{j}\right)^{2}, \\
& \sigma_{2}^{2}=b_{\alpha}^{2} \sum_{j=1}^{\infty} \Gamma_{j}^{-2 / \alpha} \dot{f}\left(0, U_{j}\right)^{2}, \\
& \sigma_{12}=b_{\alpha}^{2} \sum_{j=1}^{\infty} \Gamma_{j}^{-2 / \alpha} f\left(0, U_{j}\right) \dot{f}\left(0, U_{j}\right) .
\end{aligned}
$$


Let $h_{\rho}$ be the density function of a standardized normal random vector with covariance $\rho,|\rho|<1$. That is,

$$
h_{\rho}(x, y)=\frac{1}{2 \pi \sqrt{1-\rho^{2}}} \exp \left(-\frac{x^{2}-2 \rho x y+y^{2}}{2\left(1-\rho^{2}\right)}\right) .
$$

We conclude then that

$$
h(\lambda)=E \sigma_{1}^{-1} \sigma_{2}^{-1} \int_{0}^{\infty} y h_{\rho}\left(\lambda / \sigma_{1}, y / \sigma_{2}\right) d y
$$

with

$$
\rho=\sigma_{12} /\left(\sigma_{1} \sigma_{2}\right)
$$

and so

$$
h(\lambda)=E\left(\sigma_{2} / \sigma_{1}\right) \int_{0}^{\infty} y h_{\rho}\left(\lambda / \sigma_{1}, y\right) d y .
$$

Easy manipulations of the integral show that for any $\lambda \geq 0$,

$$
\begin{aligned}
\int_{0}^{\infty} y h_{\rho}(\lambda, y) d y= & \frac{\sqrt{1-\rho^{2}}}{2 \pi} \exp \left(-\frac{\lambda^{2}}{2\left(1-\rho^{2}\right)}\right) \\
& +\frac{\lambda \rho}{2 \pi} \exp \left(-\frac{\lambda^{2}}{2}\right) \int_{-\lambda \rho / \sqrt{1-\rho^{2}}}^{\infty} \exp \left(-\frac{y^{2}}{2}\right) d y .
\end{aligned}
$$

In particular,

$$
h(\lambda) \leq C E\left(\sigma_{2} / \sigma_{1}\right) .
$$

Therefore, both (M2) and (M3) will follow from (3.6) and (3.7) once we show that

$$
E\left(\sigma_{2} / \sigma_{1}\right)<\infty .
$$

Now, (3.8) is trivial for $1<\alpha<2$ (recall that all negative moments of a positive $\alpha / 2$-stable random variable are finite). In the case $0<\alpha \leq 1$, it follows from (1.10) and (1.11) that $\sigma_{2}^{2}$ cannot have a component independent of $\sigma_{1}^{2}$ [in the sense that the spectral measure of the $\alpha / 2$-stable random vector $\left(\sigma_{1}^{2}, \sigma_{2}^{2}\right)$ cannot have atoms at the points $(0,1)$ and $\left.(0,-1)\right]$, and so we can write

$$
b_{\alpha}^{-2}\left(\sigma_{1}^{2}, \sigma_{2}^{2}\right)={ }_{d} \beta^{2 / \alpha}\left(\sum_{j=1}^{\infty} \Gamma_{j}^{-2 / \alpha}, \sum_{j=1}^{\infty} \Gamma_{j}^{-2 / \alpha} R_{j}^{2}\right)
$$

where

$$
\beta=E\left|f\left(0, U_{1}\right)\right|^{\alpha},
$$


and $R_{j}, j \geq 1$, is a sequence of i.i.d. random variables, independent of $\Gamma_{j}$ 's, such that

$$
R_{1}={ }_{d} \dot{f}(0, W) / f(0, W),
$$

where $W$ has a distribution which is absolutely continuous with respect to the law of $U_{1}$, with Radon-Nikodym derivative given by

$$
\frac{d F_{W}}{d F_{U_{1}}}(x)=\beta^{-1}|f(0, x)|^{\alpha}
$$

The representation (3.9) follows, for example, from Corollary 3.10.2 and Property 3.2.1 of [19]. The passage from the representation (3.5) to the representation (3.9) is a version of the change of variables described in Proposition 3.5.5 in [19]. We will use such a change of variables a number of times below without further comment.

Observe that in the case $0<\alpha<1$, by (1.11),

$$
E\left|R_{1}\right|=\beta^{-1} \int_{S} \frac{|\dot{f}(0, x)|}{|f(0, x)|^{1-\alpha}} m(d x)<\infty
$$

(and the same is trivially true if $1 \leq \alpha<2$ ). Moreover, if $\alpha=1$, it follows from (1.10) that

$$
E\left|R_{1} \log \left(1+\left|R_{1}\right|\right)\right|=\beta^{-1} \int_{S}\left|\dot{f}(0, x) \log \left(1+\left|\frac{\dot{f}(0, x)}{f(0, x)}\right|\right)\right| m(d x)<\infty .
$$

Therefore, (3.8) follows from Lemma 2.1(iii) for all $0<\alpha \leq 1$, and so for all $0<\alpha<2$, which establishes (M2) and (M3).

Condition (M4) follows from (1.9) in exactly the same way as condition (M1) followed from (1.8).

It remains to check the conditions (M5), (M6) and (M7). To this end, we start by noticing that the same argument as in (3.6) and (3.7) gives us

$$
\begin{aligned}
h_{t, n}(\lambda)=E\left[\frac { \sigma _ { 2 } ( n ) } { \sigma _ { 1 } ( t , n ) } \left(\frac{\sqrt{1-\rho^{2}(t, n)}}{2 \pi} \exp \left(-\frac{\lambda^{2}}{2 \sigma_{1}^{2}(t, n)\left(1-\rho^{2}(t, n)\right)}\right)\right.\right. \\
+\frac{\lambda \rho(t, n)}{2 \pi \sigma_{1}(t, n)} \exp \left(-\lambda^{2} / 2 \sigma_{1}^{2}(t, n)\right) \\
\left.\left.\quad \times \int_{-\lambda \rho /\left(\sigma_{1}(t, n) \sqrt{1-\rho^{2}}\right)}^{\infty} \exp \left(-\frac{y^{2}}{2}\right) d y\right)\right] .
\end{aligned}
$$


Here, in parallel to (3.5),

$$
\begin{gathered}
\sigma_{1}^{2}(t, n)=b_{\alpha}^{2} \sum_{j=1}^{\infty} \Gamma_{j}^{-2 / \alpha}\left(2^{n} t\left(f\left(2^{-n}, U_{j}\right)-f\left(0, U_{j}\right)\right)+f\left(0, U_{j}\right)\right)^{2} \\
\sigma_{2}^{2}(n)=b_{\alpha}^{2} \sum_{j=1}^{\infty} \Gamma_{j}^{-2 / \alpha}\left(2^{n}\left(f\left(2^{-n}, U_{j}\right)-f\left(0, U_{j}\right)\right)\right)^{2} \\
\sigma_{12}(t, n)=b_{\alpha}^{2} \sum_{j=1}^{\infty} \Gamma_{j}^{-2 / \alpha}\left(2^{n} t\left(f\left(2^{-n}, U_{j}\right)-f\left(0, U_{j}\right)\right)+f\left(0, U_{j}\right)\right) \\
\times\left(2^{n}\left(f\left(2^{-n}, U_{j}\right)-f\left(0, U_{j}\right)\right)\right),
\end{gathered}
$$

and

$$
\rho(t, n)=\sigma_{12}(t, n) / \sigma_{1}(t, n) \sigma_{2}(n) .
$$

We claim that there is a positive number $\delta$ and an $M<\infty$ such that for all $n$ large enough and $t \in\left[0,2^{-n}\right)$,

$$
E\left(\frac{\sigma_{2}(n)}{\sigma_{1}(t, n)}\right)^{1+\delta} \leq M
$$

Before proving (3.13) note that, once proved, it will imply (M5) and (M6) in the same way as (3.8) implies (M2) and (M3). The following argument also shows that (3.13) implies (M7). Since our process is absolutely continuous, for every $\omega \in \Omega$ it is differentiable for almost every $t \in \mathfrak{R}$, and its derivative there is equal to $\dot{X}(t)$. By Fubini's theorem, for almost every $t \in \Re$,

$$
P\left(\lim _{n \rightarrow \infty} \frac{X\left(t+a_{n}\right)-X(t)}{a_{n}}=\dot{X}(t)\right)=1
$$

for every sequence of nonzero numbers $a_{n}, n \geq 1$, that converges to 0 . Since $\{X(t), t \in \mathfrak{R}\}$ is also stationary, (3.14) must hold for every $t \in \mathfrak{R}$. This implies, in particular, that for every $t \in \Re$,

$$
\frac{f\left(t+a_{n}, \cdot\right)-f(t, \cdot)}{a_{n}} \rightarrow \dot{f}(t, \cdot)
$$

in $L^{\alpha}(m)$ as $n \rightarrow \infty$ for every sequence $a_{n}, n \geq 1$, as above, and so

$$
\frac{f(t+h, \cdot)-f(t, \cdot)}{h} \rightarrow \dot{f}(t, \cdot)
$$

as $h \rightarrow 0$. Therefore, for every sequence $t_{n} \in\left(0,2^{-n}\right], n \geq 1$, we have, in $L^{\alpha / 2}(m)$,

$$
\begin{aligned}
&\left(2^{n} t_{n}\left(f\left(2^{-n}, \cdot\right)-f(0, \cdot)\right)+f(0, \cdot)\right)^{2} \rightarrow(f(0, \cdot))^{2} \\
&\left(2^{n}\left(f\left(2^{-n}, \cdot\right)-f(0, \cdot)\right)\right)^{2} \rightarrow(\dot{f}(0, \cdot))^{2} \\
&\left(2^{n} t_{n}\left(f\left(2^{-n}, \cdot\right)-f(0, \cdot)\right)+f(0, \cdot)\right) \\
& \times\left(2^{n}\left(f\left(2^{-n}, \cdot\right)-f(0, \cdot)\right)\right) \rightarrow f(0, \cdot) \dot{f}(0, \cdot)
\end{aligned}
$$


as $n \rightarrow \infty$. Therefore, the sequence $V_{n}=\left(\sigma_{1}^{2}\left(t_{n}, n\right), \sigma_{2}^{2}(n), \sigma_{12}\left(t_{n}, n\right)\right), n \geq 1$, of $\alpha / 2$-stable random vectors in $\Re^{3}$, defined by (3.12), converges in probability, as $n \rightarrow \infty$, to the $\alpha / 2$-stable random vector $V=\left(\sigma_{1}^{2}, \sigma_{2}^{2}, \sigma_{12}\right)$ defined by (3.5). It follows from (3.6), (3.7) and (3.11) that for every $\lambda \geq 0$,

$$
\begin{gathered}
h_{t, n}(\lambda)=E \Phi\left(V_{n}\right), \\
h(\lambda)=E \Phi(V),
\end{gathered}
$$

where $\Phi$ is a measurable function, from $\mathfrak{R}^{3} \rightarrow \mathfrak{R}$, with a negligible set of discontinuities (with respect to the distribution of $V$ ). Therefore, by the continuous mapping theorem,

$$
\Phi\left(V_{n}\right) \Rightarrow \Phi(V)
$$

as $n \rightarrow \infty$. However, (3.13) implies that the sequence $\Phi\left(V_{n}\right), n \geq 1$ is uniformly integrable, which implies (M7). Therefore, to complete the proof of the theorem we only have to check (3.13).

Suppose first that $1<\alpha<2$. Choose $\delta=\alpha^{1 / 2}-1$. Then, by Hölder's inequality,

$$
\begin{aligned}
& E\left(\frac{\sigma_{2}(n)}{\sigma_{1}(t, n)}\right)^{1+\delta} \\
& \quad=E\left(\frac{\sigma_{2}(n)}{\sigma_{1}(t, n)}\right)^{\alpha^{1 / 2}} \\
& \quad \leq\left(E \sigma_{2}(n)^{\alpha^{3 / 4}}\right)^{\alpha^{-1 / 4}}\left(E \sigma_{1}(t, n)^{-\alpha^{1 / 2} /\left(1-\alpha^{-1 / 4}\right)}\right)^{1-\alpha^{-1 / 4}} \\
& \quad \leq C\left\|\frac{f\left(2^{-n}, \cdot\right)-f(0, \cdot)}{2^{-n}}\right\|_{\alpha}^{\alpha^{1 / 2}}\left\|2^{n} t\left(f\left(2^{-n}, \cdot\right)-f(0, \cdot)\right)+f(0, \cdot)\right\|_{\alpha}^{-\alpha^{1 / 2}} \\
& \quad \leq M
\end{aligned}
$$

for some finite constant $M$ by (3.16), where $C$ is also a finite positive constant. This proves (3.13) in the case $1<\alpha<2$.

We turn now to the case $0<\alpha \leq 1$. It follows from (1.12) that $\sigma_{2}^{2}(n)$ cannot have a component independent of $\sigma_{1}^{2}(t, n)$, and so similarly to (3.9) we obtain

$$
b_{\alpha}^{-2}\left(\sigma_{1}^{2}(t, n), \sigma_{2}^{2}(n)\right)={ }_{d} \gamma_{t, n}^{2 / \alpha}\left(\sum_{j=1}^{\infty} \Gamma_{j}^{-2 / \alpha}, \sum_{j=1}^{\infty} \Gamma_{j}^{-2 / \alpha} T_{j}^{2}\right),
$$

where

$$
\gamma_{t, n}=E\left|2^{n} t\left(f\left(2^{-n}, U_{1}\right)-f\left(0, U_{1}\right)\right)+f\left(0, U_{1}\right)\right|^{\alpha}
$$


and $T_{j}, j \geq 1$, is now a sequence of i.i.d. random variables, independent of the Poisson arrivals such that

$$
T_{1}={ }_{d} \frac{2^{n}\left(f\left(2^{-n}, V\right)-f(0, V)\right)}{2^{n} t\left(f\left(2^{-n}, V\right)-f(0, V)\right)+f(0, V)},
$$

where $V$ has a distribution which is absolutely continuous with respect to the law of $U_{1}$, with Radon-Nikodym derivative given by

$$
\frac{d F_{V}}{d F_{U_{1}}}(x)=\gamma_{t, n}^{-1}\left|2^{n} t\left(f\left(2^{-n}, x\right)-f(0, x)\right)+f(0, x)\right|^{\alpha} .
$$

Observe that for all $n$ big enough and $t \in\left(0,2^{-n}\right]$,

$$
\gamma_{t, n} \geq E\left|f\left(0, U_{1}\right)\right|^{\alpha} / 2>0 .
$$

Therefore, with $\delta$ as in (1.12) and for all $n$ and $t$ as above, we have

$$
\begin{aligned}
E\left|T_{1}\right|^{1+\delta} & =\gamma_{t, n}^{-1} \int_{S} \frac{\left|2^{n}\left(f\left(2^{-n}, x\right)-f(0, x)\right)\right|^{1+\delta}}{\left|2^{n} t\left(f\left(2^{-n}, x\right)-f(0, x)\right)+f(0, x)\right|^{1+\delta-\alpha}} m(d x) \\
& \leq 2 M / E\left|f\left(0, U_{1}\right)\right|^{\alpha}<\infty,
\end{aligned}
$$

and so (3.13) in the case $0<\alpha \leq 1$ follows from Lemma 2.1(iii).

The integral formula (1.7) is, therefore, proved completely in the case when the control measure $m$ of the $S \alpha S$ random measure $M$ is a probability measure. In the general case of a $\sigma$-finite control measure $m$, let $\eta$ be a probability measure on $S$ equivalent to $m$. Let $b(x)=(d m / d \eta)(x), x \in S$. Then we can represent the process $\{X(t), t \in \mathfrak{R}\}$ and its derivative $\{\dot{X}(t)$, $t \in \mathfrak{R}\}$ as in (1.1) and (1.6), with $m$ replaced by $\eta, f(t, x)$ replaced by $\tilde{f}(t, x)=f(t, x) b(x)^{1 / \alpha}$ and $\dot{f}(t, x)$ replaced by $\tilde{f}(t, x)=\dot{f}(t, x) b(x)^{1 / \alpha}$. Clearly, $\tilde{f}, \tilde{f}$ and $\eta$ satisfy the conditions of the theorem. Therefore, the integral formula (1.7) holds in the general case as well.

This completes the main part of the proof of the theorem. What remains is to show the veracity of the asymptotic formula (1.13). This, however, follows from Proposition 4.1 of the following section, with $g_{1}(x)=f(0, x)$ and $g_{2}(x)=\dot{f}(0, x), x \in S$. Observe that (4.3) and (4.4) follow from (1.11) and (1.10), correspondingly. Therefore, (1.13) is an immediate consequence of the above proposition, and the proof of the theorem is complete.

REMARK. Inspecting the assumptions of Proposition 4.1 in the next section shows that one cannot dispense with conditions (1.10) and (1.11) in Theorem 1.1. Condition (1.12) can, on the other hand, most likely be relaxed (and, in 
particular cases, replaced by neater conditions), either by using an argument different from the uniform integrability one in our proof, or just by using a set of conditions different from those given in Marcus [11]. In other words, the conclusion (1.13) remains true whenever (1.7), (1.10) and (1.11) hold. In particular, (1.13) holds under (1.10) and (1.11) for continuously differentiable stationary processes, by the result of Michna and Rychlik [14].

4. The asymptotic behavior of an integral. In this section we deal with the second step in the proof of Theorem 1.1-the asymptotic behavior of the integral in the right-hand side of (1.7). This is described in Proposition 4.1. This proposition may be of independent interest, and so our notation in this section is to a certain degree independent of that of the previous section, which is tied in more closely to the specific application of Proposition 4.1 there.

Let $(U, W)$ be a nondegenerate (i.e., not concentrated on any proper subspace of $\Re^{2}$ ) $S \alpha S$ random vector, $0<\alpha<2$, given in the form

$$
\begin{gathered}
U=\int_{S} g_{1}(x) M(d x), \\
W=\int_{S} g_{2}(x) M(d x),
\end{gathered}
$$

where $M$ is, as before, an $S \alpha S$ random measure on $S$ with a control measure $m$, and $g_{i} \in L^{\alpha}(m), i=1,2$. We denote the joint density of $U$ and $W$ by $f_{U, W}$ and the marginal densities by $f_{U}$ and $f_{W}$ accordingly. Let

$$
I(\lambda)=\int_{0}^{\infty} y f_{U, W}(\lambda, y) d y, \quad \lambda \geq 0 .
$$

The main result of this section is the asymptotic behavior of $I(\lambda)$ as $\lambda \rightarrow \infty$.

Proposition 4.1. Let $I(\lambda)$ be given by (4.2). If $0<\alpha<1$, assume that

$$
\int_{S}\left|g_{2}(x)\right|\left|g_{1}(x)\right|^{\alpha-1} m(d x)<\infty,
$$

while in the case $\alpha=1$ assume that

$$
\int_{S}\left|g_{2}(x)\right|\left|\log \left(1+\left|g_{2}(x)\right|^{2} /\left.g_{1}(x)\right|^{2}\right)\right| m(d x)<\infty .
$$

(No extra assumptions are needed for $1<\alpha<2$.) Then $I(\lambda)<\infty$ for all $\lambda \geq 0$, and

$$
\lim _{\lambda \rightarrow \infty} \lambda^{\alpha} I(\lambda)=\alpha 2^{\alpha-1 / 2} \pi^{-(1+1 / \alpha) / 2}\left(\Gamma\left(\frac{\alpha+1}{2}\right)\right)^{1+1 / \alpha} C_{\alpha}^{-1 / \alpha} \kappa_{\alpha}\left(g_{1}, g_{2}\right),
$$

where $C_{\alpha}$ is given by (2.11) and

$$
\kappa_{\alpha}\left(g_{1}, g_{2}\right)=\int_{S}\left|g_{2}(x)\right|\left|g_{1}(x)\right|^{\alpha-1} \mathbf{1}\left(g_{1}(x) g_{2}(x)>0\right) m(d x),
$$

where here and below $\mathbf{1}(\cdot)$ is the usual indicator function. 
Proof. Obviously,

$$
I(\lambda) \leq f_{U}(\lambda) E(|W| \mid U=\lambda) .
$$

The right-hand side of (4.7) is clearly finite if $1<\alpha<2$. In the case $0<\alpha<1$ the finiteness of the right-hand side of (4.7) under assumption (4.3) follows from [4], while in the case $\alpha=1$, the finiteness of the right-hand side of (4.7) under the assumption (4.4) follows from [5].

Assume for the moment that the control measure $m$ of the $S \alpha S$ random measure $M$ in (4.1) is actually a probability measure on $S$. Then, as we have done before, one can represent the random vector $(U, W)$ in the form

$$
\begin{aligned}
& U=b_{\alpha} \sum_{j=1}^{\infty} G_{j} \Gamma_{j}^{-1 / \alpha} g_{1}\left(U_{j}\right), \\
& W=b_{\alpha} \sum_{j=1}^{\infty} G_{j} \Gamma_{j}^{-1 / \alpha} g_{2}\left(U_{j}\right)
\end{aligned}
$$

(the representation is, of course, in distribution), where $b_{\alpha}$ is given by (3.4), and where, as before, $G_{j}, j \geq 1, \Gamma_{j}, j \geq 1$, and $U_{j}, j \geq 1$, are three independent sequences of random variables, such that $G_{j}, j \geq 1$, are i.i.d. standard normal random variables, $\Gamma_{j}, j \geq 1$, are the arrival times of a unit rate Poisson process on $(0, \infty)$ and $U_{j}, j \geq 1$, are i.i.d. $S$-valued random variables with a common law $m$. We have seen before that, conditionally on $\Gamma_{j}, j \geq 1$, and $U_{j}, j \geq 1$, the random variables $U$ and $W$ are jointly normal, with zero means and a variance-covariance matrix with elements

$$
\begin{aligned}
& \sigma_{1}^{2}=b_{\alpha}^{2} \sum_{j=1}^{\infty} \Gamma_{j}^{-2 / \alpha} g_{1}\left(U_{j}\right)^{2}, \\
& \sigma_{2}^{2}=b_{\alpha}^{2} \sum_{j=1}^{\infty} \Gamma_{j}^{-2 / \alpha} g_{2}\left(U_{j}\right)^{2}, \\
& \sigma_{12}=b_{\alpha}^{2} \sum_{j=1}^{\infty} \Gamma_{j}^{-2 / \alpha} g_{1}\left(U_{j}\right) g_{2}\left(U_{j}\right) .
\end{aligned}
$$

Again, using the technique developed in the previous section, we conclude by (3.6) and (3.7) that

$$
\begin{aligned}
I(\lambda)= & E\left(\frac{\sigma_{2}}{\sigma_{1}} \frac{\sqrt{1-\rho^{2}}}{2 \pi} \exp \left(-\frac{\lambda^{2}}{2 \sigma_{1}^{2}\left(1-\rho^{2}\right)}\right)\right) \\
& +E\left(\frac{\sigma_{2} \rho}{2 \pi \sigma_{1}^{2}} \lambda \exp \left(-\frac{\lambda^{2}}{2 \sigma_{1}^{2}}\right) \int_{-\lambda \rho /\left(\sigma_{1} \sqrt{1-\rho^{2}}\right)}^{\infty} \exp \left(-\frac{y^{2}}{2}\right) d y\right) \\
= & E\left(\frac{\sigma_{2}}{\sigma_{1}} \frac{\sqrt{1-\rho^{2}}}{2 \pi} \exp \left(-\frac{\lambda^{2}}{2 \sigma_{1}^{2}\left(1-\rho^{2}\right)}\right)\right) \\
(4.10)+ & E\left(\frac{\sigma_{2} \rho}{\sqrt{2 \pi} \sigma_{1}^{2}} \lambda \exp \left(-\frac{\lambda^{2}}{2 \sigma_{1}^{2}}\right) \mathbf{1}(\rho>0)\right)
\end{aligned}
$$




$$
\begin{aligned}
& \quad+E\left(\frac{\sigma_{2} \rho}{2 \pi \sigma_{1}^{2}} \lambda \exp \left(-\frac{\lambda^{2}}{2 \sigma_{1}^{2}}\right) \int_{-\lambda \rho /\left(\sigma_{1} \sqrt{1-\rho^{2}}\right)}^{\infty} \exp \left(-\frac{y^{2}}{2}\right) d y \mathbf{1}(\rho \leq 0)\right) \\
& \quad-E\left(\frac{\sigma_{2} \rho}{2 \pi \sigma_{1}^{2}} \lambda \exp \left(-\frac{\lambda^{2}}{2 \sigma_{1}^{2}}\right) \int_{-\infty}^{-\lambda \rho /\left(\sigma_{1} \sqrt{1-\rho^{2}}\right)} \exp \left(-\frac{y^{2}}{2}\right) d y \mathbf{1}(\rho>0)\right) \\
& :=\sum_{i=1}^{4} E C_{i}(\lambda) .
\end{aligned}
$$

We will prove that

$$
\begin{aligned}
& \lim _{\lambda \rightarrow \infty} \lambda^{\alpha} E C_{2}(\lambda) \\
& \quad=\alpha 2^{\alpha-1 / 2} \pi^{-(1+1 / \alpha) / 2}\left(\Gamma\left(\frac{\alpha+1}{2}\right)\right)^{1+1 / \alpha} C_{\alpha}^{-1 / \alpha} \kappa_{\alpha}\left(g_{1}, g_{2}\right),
\end{aligned}
$$

and

$$
\lim _{\lambda \rightarrow \infty} \lambda^{\alpha} E C_{i}(\lambda)=0
$$

for $i=1,3,4$, which will imply the conclusion of the proposition by (4.10).

We start with the proof of (4.11). We have

$$
E C_{2}(\lambda)=\frac{1}{\sqrt{2 \pi}} \lambda E\left(\frac{\sigma_{12}}{\sigma_{1}^{3}} \exp \left(-\frac{\lambda^{2}}{2 \sigma_{1}^{2}}\right) \mathbf{1}\left(\sigma_{12}>0\right)\right):=\frac{1}{\sqrt{2 \pi}} \lambda H_{1}(\lambda),
$$

and so the proof of (4.11) reduces to proving that

$$
\lim _{\lambda \rightarrow \infty} \lambda^{\alpha+1} H_{1}(\lambda)=\alpha 2^{\alpha} \pi^{-1 / 2 \alpha}\left(\Gamma\left(\frac{\alpha+1}{2}\right)\right)^{1+1 / \alpha} C_{\alpha}^{-1 / \alpha} \kappa_{\alpha}\left(g_{1}, g_{2}\right) .
$$

Observe that by (4.9)

$$
\begin{array}{r}
H_{1}(\lambda)=b_{\alpha}^{-1} E\left(\frac{\sum_{j=1}^{\infty} \Gamma_{j}^{-2 / \alpha} g_{1}\left(U_{j}\right) g_{2}\left(U_{j}\right)}{\left(\sum_{j=1}^{\infty} \Gamma_{j}^{-2 / \alpha} g_{1}\left(U_{j}\right)^{2}\right)^{3 / 2}} \exp \left(-\frac{\lambda^{2}}{2 \sum_{j=1}^{\infty} \Gamma_{j}^{-2 / \alpha} g_{1}\left(U_{j}\right)^{2}}\right)\right. \\
\left.\times \mathbf{1}\left(\sum_{j=1}^{\infty} \Gamma_{j}^{-2 / \alpha} g_{1}\left(U_{j}\right) g_{2}\left(U_{j}\right)>0\right)\right) .
\end{array}
$$

Furthermore, we can change the representation in (4.9) in the same way as was done in the proof of Theorem 1.1 to obtain

$$
b_{\alpha}^{-2}\left(\sigma_{1}^{2}, \sigma_{12}\right)=_{d} \beta^{2 / \alpha}\left(\sum_{j=1}^{\infty} \Gamma_{j}^{-2 / \alpha}, \sum_{j=1}^{\infty} \Gamma_{j}^{-2 / \alpha} R_{j}\right),
$$

where this time

$$
\beta=E\left|g_{1}\left(U_{1}\right)\right|^{\alpha}
$$


and $R_{j}, j \geq 1$, is an independent of the Poisson arrivals sequence of i.i.d. random variables, such that

$$
R_{1}={ }_{d} g_{2}(W) / g_{1}(W),
$$

where $W$ has a distribution which is absolutely continuous with respect to the law of $U_{1}$, with the Radon-Nikodym derivative given by

$$
\frac{d F_{W}}{d F_{U_{1}}}(x)=\beta^{-1}\left|g_{1}(x)\right|^{\alpha} .
$$

Once again, we have

$$
E\left|R_{1}\right|=\beta^{-1} E\left|g_{2}(U)\right|\left|g_{1}(U)\right|^{\alpha-1}<\infty .
$$

(This is trivial for $1 \leq \alpha<2$ and follows from (4.3) if $0<\alpha<1$.)

Therefore,

$$
\begin{aligned}
H_{1}(\lambda)=b_{\alpha}^{-1} \beta^{-1 / \alpha} E\left(\frac{\sum_{j=1}^{\infty} \Gamma_{j}^{-2 / \alpha} R_{j}}{\left(\sum_{j=1}^{\infty} \Gamma_{j}^{-2 / \alpha}\right)^{3 / 2}} \exp (\right. & \left.-\frac{\lambda^{2}}{2 \beta^{2 / \alpha} \sum_{j=1}^{\infty} \Gamma_{j}^{-2 / \alpha}}\right) \\
& \left.\times \mathbf{1}\left(\sum_{j=1}^{\infty} \Gamma_{j}^{-2 / \alpha} R_{j}>0\right)\right) \\
:=b_{\alpha}^{-1} \beta^{-1 / \alpha} E Z . &
\end{aligned}
$$

We now decompose $H_{1}(\lambda)$ in the following way. For a $(1 \vee \alpha)<\theta<2$ and $0<\varepsilon<1$ write

$$
\begin{aligned}
H_{1}(\lambda)= & b_{\alpha}^{-1} \beta^{-1 / \alpha} E\left(Z \mathbf{1}\left(\sum_{j=1}^{\infty} \Gamma_{j}^{-2 / \alpha} \leq \lambda^{\theta}\right)\right) \\
& +b_{\alpha}^{-1} \beta^{-1 / \alpha} E\left(Z \mathbf{1}\left(\sum_{j=1}^{\infty} \Gamma_{j}^{-2 / \alpha}>\lambda^{\theta}, \frac{\Gamma_{1}^{-2 / \alpha}}{\sum_{j=1}^{\infty} \Gamma_{j}^{-2 / \alpha}} \leq 1-\varepsilon\right)\right) \\
& +b_{\alpha}^{-1} \beta^{-1 / \alpha} E\left(Z \mathbf{1}\left(\sum_{j=1}^{\infty} \Gamma_{j}^{-2 / \alpha}>\lambda^{\theta}, \frac{\Gamma_{1}^{-2 / \alpha}}{\sum_{j=1}^{\infty} \Gamma_{j}^{-2 / \alpha}}>1-\varepsilon\right)\right) \\
:= & H_{11}(\lambda)+H_{12}(\lambda)+H_{13}(\lambda) .
\end{aligned}
$$

In the sequel we will be using, in addition to (2.12), the following elementary observation. For any $p>0, \theta<2$ and $\lambda>(2 p)^{1 /(2-\theta)}$,

$$
\sup _{0<z \leq \lambda^{\theta}} z^{-p} \exp \left(-\lambda^{2} / 2 z\right)=\lambda^{-\theta p} \exp \left(-\lambda^{2-\theta} / 2\right) .
$$


Using (4.20) we immediately conclude that for all $\lambda$ big enough

$$
\begin{aligned}
H_{11}(\lambda) \leq b_{\alpha}^{-1} \beta^{-1 / \alpha} E\left|R_{1}\right| E\left(\frac{1}{\left(\sum_{j=1}^{\infty} \Gamma_{j}^{-2 / \alpha}\right)^{1 / 2}} \exp (\right. & \left.-\frac{\lambda^{2}}{2 \beta^{2 / \alpha} \sum_{j=1}^{\infty} \Gamma_{j}^{-2 / \alpha}}\right) \\
& \left.\times \mathbf{1}\left(\sum_{j=1}^{\infty} \Gamma_{j}^{-2 / \alpha} \leq \lambda^{\theta}\right)\right) \\
& \leq C \lambda^{-\theta / 2} \exp \left(-\lambda^{2-\theta} / C\right),
\end{aligned}
$$

where $C$ is a finite positive constant that in the sequel may be expected to change from line to line. We see that

$$
\lim _{\lambda \rightarrow \infty} \lambda^{\alpha+1} H_{11}(\lambda)=0 .
$$

Let

$$
A=\left\{\sum_{j=1}^{\infty} \Gamma_{j}^{-2 / \alpha}>\lambda^{\theta}, \frac{\Gamma_{1}^{-2 / \alpha}}{\sum_{j=1}^{\infty} \Gamma_{j}^{-2 / \alpha}} \leq 1-\varepsilon\right\} .
$$

Observe that by Lemma 2.1(i)

$$
P(A) \leq P\left(\sum_{j=2}^{\infty} \Gamma_{j}^{-2 / \alpha}>\varepsilon \lambda^{\theta}\right) \leq C \lambda^{-\theta \min (1, \alpha)},
$$

and so

$$
\begin{aligned}
H_{12}(\lambda) & \leq b_{\alpha}^{-1} \beta^{-1 / \alpha} E\left|R_{1}\right| E\left(\frac{1}{\left(\sum_{j=1}^{\infty} \Gamma_{j}^{-2 / \alpha}\right)^{1 / 2}} \exp \left(-\frac{\lambda^{2}}{2 \beta^{2 / \alpha} \sum_{j=1}^{\infty} \Gamma_{j}^{-2 / \alpha}}\right) \mathbf{1}_{A}\right) \\
& \leq C\left(E\left(\frac{1}{\sum_{j=1}^{\infty} \Gamma_{j}^{-2 / \alpha}} \exp \left(-\frac{\lambda^{2}}{\beta^{2 / \alpha} \sum_{j=1}^{\infty} \Gamma_{j}^{-2 / \alpha}}\right)\right)\right)^{1 / 2}(P(A))^{1 / 2} \\
& \leq C \lambda^{-(\alpha+2) / 2} \lambda^{-\theta \min (1, \alpha) / 2},
\end{aligned}
$$

by Lemma 2.2(i). Since $\theta>1 \vee \alpha$, we obtain immediately that

$$
\lim _{\lambda \rightarrow \infty} \lambda^{\alpha+1} H_{12}(\lambda)=0 \text {. }
$$

We conclude by (4.21) and (4.22) that (4.13) reduces to proving that

$$
\lim _{\lambda \rightarrow \infty} \lambda^{\alpha+1} H_{13}(\lambda)=\alpha 2^{\alpha} \pi^{-1 / 2 \alpha}\left(\Gamma\left(\frac{\alpha+1}{2}\right)\right)^{1+1 / \alpha} C_{\alpha}^{-1 / \alpha} \kappa_{\alpha}\left(g_{1}, g_{2}\right) .
$$

We further decompose $H_{13}$ as follows. Set

$$
B=\left\{\sum_{j=1}^{\infty} \Gamma_{j}^{-2 / \alpha} R_{j}>0, \sum_{j=1}^{\infty} \Gamma_{j}^{-2 / \alpha}>\lambda^{\theta}, \frac{\Gamma_{1}^{-2 / \alpha}}{\sum_{j=1}^{\infty} \Gamma_{j}^{-2 / \alpha}}>1-\varepsilon\right\} .
$$


We have

$$
\begin{aligned}
H_{13}(\lambda)= & b_{\alpha}^{-1} \beta^{-1 / \alpha} E\left(\frac{\Gamma_{1}^{-2 / \alpha} R_{1}}{\left(\sum_{j=1}^{\infty} \Gamma_{j}^{-2 / \alpha}\right)^{3 / 2}} \exp \left(-\frac{\lambda^{2}}{2 \beta^{2 / \alpha} \sum_{j=1}^{\infty} \Gamma_{j}^{-2 / \alpha}}\right) \mathbf{1}_{B}\right) \\
& \quad+b_{\alpha}^{-1} \beta^{-1 / \alpha} E\left(\frac{\sum_{j=2}^{\infty} \Gamma_{j}^{-2 / \alpha} R_{j}}{\left(\sum_{j=1}^{\infty} \Gamma_{j}^{-2 / \alpha}\right)^{3 / 2}} \exp \left(-\frac{\lambda^{2}}{2 \beta^{2 / \alpha} \sum_{j=1}^{\infty} \Gamma_{j}^{-2 / \alpha}}\right) \mathbf{1}_{B}\right) \\
:= & H_{131}(\lambda)+H_{132}(\lambda) .
\end{aligned}
$$

We will see that $H_{131}(\lambda)$ is the main term in (4.23). We start by showing that $H_{132}(\lambda)$ is small. Specifically, we claim that there is a $C \in(0, \infty)$ such that

$$
\limsup _{\lambda \rightarrow \infty} \lambda^{\alpha+1}\left|H_{132}(\lambda)\right| \leq C \varepsilon
$$

Indeed,

$$
\begin{aligned}
\left|H_{132}(\lambda)\right| & \leq C E\left|R_{1}\right| E\left(\frac{\sum_{j=2}^{\infty} \Gamma_{j}^{-2 / \alpha}}{\left(\sum_{j=1}^{\infty} \Gamma_{j}^{-2 / \alpha}\right)^{3 / 2}} \exp \left(-\frac{\lambda^{2}}{2 \beta^{2 / \alpha} \sum_{j=1}^{\infty} \Gamma_{j}^{-2 / \alpha}}\right)\right. \\
& \left.\times \mathbf{1}\left(\Gamma_{1}^{-2 / \alpha}>(1-\varepsilon) \sum_{j=1}^{\infty} \Gamma_{j}^{-2 / \alpha}\right)\right) \\
& \leq C \varepsilon E\left(\frac{1}{\left(\sum_{j=1}^{\infty} \Gamma_{j}^{-2 / \alpha}\right)^{1 / 2}} \exp \left(-\frac{\lambda^{2}}{2 \beta^{2 / \alpha} \sum_{j=1}^{\infty} \Gamma_{j}^{-2 / \alpha}}\right)\right) \\
& \leq C \varepsilon \lambda^{-(\alpha+1)}
\end{aligned}
$$

once again by Lemma 2.2(i). This establishes (4.25).

We now consider $H_{131}(\lambda)$, which we decompose once more. Let

$$
Z_{1}=b_{\alpha}^{-1} \beta^{-1 / \alpha} \frac{\Gamma_{1}^{-2 / \alpha} R_{1}}{\left(\sum_{j=1}^{\infty} \Gamma_{j}^{-2 / \alpha}\right)^{3 / 2}} \exp \left(-\frac{\lambda^{2}}{2 \beta^{2 / \alpha} \sum_{j=1}^{\infty} \Gamma_{j}^{-2 / \alpha}}\right) .
$$

For a $\delta \in(0,1)$ write

$$
\begin{aligned}
H_{131}(\lambda) & =E\left(Z_{1} \mathbf{1}_{B} \mathbf{1}\left(\left|R_{1}\right|>\delta\right)\right)+E\left(Z_{1} \mathbf{1}_{B} \mathbf{1}\left(\left|R_{1}\right| \leq \delta\right)\right) \\
& :=\bar{H}_{1}(\lambda)+\bar{H}_{2}(\lambda)
\end{aligned}
$$

Note that, as before,

$$
\begin{aligned}
& \left|\bar{H}_{2}(\lambda)\right| \\
& \quad \leq C E\left(\left|R_{1}\right| \mathbf{1}\left(\left|R_{1}\right| \leq \delta\right)\right) E\left(\frac{1}{\left(\sum_{j=1}^{\infty} \Gamma_{j}^{-2 / \alpha}\right)^{1 / 2}} \exp \left(-\frac{\lambda^{2}}{2 \beta^{2 / \alpha} \sum_{j=1}^{\infty} \Gamma_{j}^{-2 / \alpha}}\right)\right) \\
& \quad \leq C h(\delta) \lambda^{-(\alpha+1)},
\end{aligned}
$$


where $h(\delta)=E\left(\left|R_{1}\right| \mathbf{1}\left(\left|R_{1}\right| \leq \delta\right)\right)$ and $h(\delta) \rightarrow 0$ as $\delta \rightarrow 0$. Therefore,

$$
\limsup _{\lambda \rightarrow \infty} \lambda^{\alpha+1}\left|\bar{H}_{2}(\lambda)\right| \leq C h(\delta) \text {. }
$$

Consider now $\bar{H}_{1}$ and write

$$
\begin{aligned}
\bar{H}_{1}(\lambda)=b_{\alpha}^{-1} \beta^{-1 / \alpha} E & \left(\frac{\Gamma_{1}^{-2 / \alpha} R_{1}}{\left(\sum_{j=1}^{\infty} \Gamma_{j}^{-2 / \alpha}\right)^{3 / 2}} \exp \left(-\frac{\lambda^{2}}{2 \beta^{2 / \alpha} \sum_{j=1}^{\infty} \Gamma_{j}^{-2 / \alpha}}\right)\right. \\
& \left.\times \mathbf{1}\left(R_{1}>\delta, \sum_{j=1}^{\infty} \Gamma_{j}^{-2 / \alpha}>\lambda^{\theta}, \Gamma_{1}^{-2 / \alpha}>(1-\varepsilon) \sum_{j=1}^{\infty} \Gamma_{j}^{-2 / \alpha}\right)\right)
\end{aligned}
$$

$$
\begin{aligned}
& +b_{\alpha}^{-1} \beta^{-1 / \alpha} E\left(\frac{\Gamma_{1}^{-2 / \alpha} R_{1}}{\left(\sum_{j=1}^{\infty} \Gamma_{j}^{-2 / \alpha}\right)^{3 / 2}} \exp \left(-\frac{\lambda^{2}}{2 \beta^{2 / \alpha} \sum_{j=1}^{\infty} \Gamma_{j}^{-2 / \alpha}}\right)\right. \\
& \quad \times \mathbf{1}\left(\sum_{j=1}^{\infty} \Gamma_{j}^{-2 / \alpha}>\lambda^{\theta}, \Gamma_{1}^{-2 / \alpha}>(1-\varepsilon) \sum_{j=1}^{\infty} \Gamma_{j}^{-2 / \alpha}\right) \\
& \quad \times\left(-\mathbf{1}\left(R_{1}>\delta\right)+\mathbf{1}\left(\left|R_{1}\right|>\delta, \sum_{j=1}^{\infty} \Gamma_{j}^{-2 / \alpha} R_{j}>0\right)\right) \\
& :=\bar{H}_{11}(\lambda)+\bar{H}_{12}(\lambda) .
\end{aligned}
$$

We claim that

$$
\limsup \lambda^{\alpha+1}\left|\bar{H}_{12}(\lambda)\right|=0 \text {. }
$$

To this end denote

$$
\begin{aligned}
Z_{2}= & b_{\alpha}^{-1} \beta^{-1 / \alpha} \frac{\Gamma_{1}^{-2 / \alpha} R_{1}}{\left(\sum_{j=1}^{\infty} \Gamma_{j}^{-2 / \alpha}\right)^{3 / 2}} \exp \left(-\frac{\lambda^{2}}{2 \beta^{2 / \alpha} \sum_{j=1}^{\infty} \Gamma_{j}^{-2 / \alpha}}\right) \\
& \times \mathbf{1}\left(\sum_{j=1}^{\infty} \Gamma_{j}^{-2 / \alpha}>\lambda^{\theta}, \Gamma_{1}^{-2 / \alpha}>(1-\varepsilon) \sum_{j=1}^{\infty} \Gamma_{j}^{-2 / \alpha}\right)
\end{aligned}
$$

and observe that

$$
\begin{aligned}
\left|\bar{H}_{12}(\lambda)\right| \leq & E\left(Z_{2} \mathbf{1}\left(R_{1}>\delta, \sum_{j=1}^{\infty} \Gamma_{j}^{-2 / \alpha} R_{j} \leq 0\right)\right) \\
& +E\left(Z_{2} \mathbf{1}\left(R_{1}<-\delta, \sum_{j=1}^{\infty} \Gamma_{j}^{-2 / \alpha} R_{j}>0\right)\right) \\
\leq & C E\left(\frac{\Gamma_{1}^{-2 / \alpha}\left|R_{1}\right|}{\left(\sum_{j=1}^{\infty} \Gamma_{j}^{-2 / \alpha}\right)^{3 / 2}} \exp \left(-\frac{\lambda^{2}}{2 \beta^{2 / \alpha} \sum_{j=1}^{\infty} \Gamma_{j}^{-2 / \alpha}}\right)\right. \\
& \left.\times \mathbf{1}\left(\sum_{j=2}^{\infty} \Gamma_{j}^{-2 / \alpha}\left|R_{j}\right|>\delta \Gamma_{1}^{-2 / \alpha}, \Gamma_{1}^{-2 / \alpha}>(1-\varepsilon) \lambda^{\theta}\right)\right)
\end{aligned}
$$




$$
\begin{array}{r}
\leq C E\left(\frac{\Gamma_{1}^{-2 / \alpha}\left|R_{1}\right|}{\left(\sum_{j=1}^{\infty} \Gamma_{j}^{-2 / \alpha}\right)^{3 / 2}} \exp \left(-\frac{\lambda^{2}}{2 \beta^{2 / \alpha} \sum_{j=1}^{\infty} \Gamma_{j}^{-2 / \alpha}}\right)\right. \\
\left.\times \mathbf{1}\left(\sum_{j=2}^{\infty} \Gamma_{j}^{-2 / \alpha}\left|R_{j}\right|>\delta(1-\varepsilon) \lambda^{\theta}\right)\right) .
\end{array}
$$

Now define

$$
B_{1}=\left\{\sum_{j=2}^{\infty} \Gamma_{j}^{-2 / \alpha}\left|R_{j}\right|>\delta(1-\varepsilon) \lambda^{\theta}\right\} .
$$

It follows once again from Lemma 2.1(i) that $P\left(B_{1}\right) \leq C \lambda^{-\theta \min (1, \alpha)}$, and so (4.29) follows at once as in (4.21).

It only remains therefore to consider $\bar{H}_{11}(\lambda)$. We have

$$
\begin{aligned}
& \bar{H}_{11}(\lambda)=b_{\alpha}^{-1} \beta^{-1 / \alpha} E\left(R_{1} \mathbf{1}\left(R_{1}>\delta\right)\right) \\
& \quad \times E\left(\frac{\Gamma_{1}^{-2 / \alpha}}{\left(\sum_{j=1}^{\infty} \Gamma_{j}^{-2 / \alpha}\right)^{3 / 2}} \exp \left(-\frac{\lambda^{2}}{2 \beta^{2 / \alpha} \sum_{j=1}^{\infty} \Gamma_{j}^{-2 / \alpha}}\right)\right. \\
& \left.\quad \times \mathbf{1}\left(\sum_{j=1}^{\infty} \Gamma_{j}^{-2 / \alpha}>\lambda^{\theta}, \Gamma_{1}^{-2 / \alpha}>(1-\varepsilon) \sum_{j=1}^{\infty} \Gamma_{j}^{-2 / \alpha}\right)\right) \\
& :=b_{\alpha}^{-1} \beta^{-1 / \alpha} E\left(R_{1} \mathbf{1}\left(R_{1}>\delta\right)\right) T(\lambda) .
\end{aligned}
$$

Observe that

$$
(1-\varepsilon) T_{+}(\lambda) \leq T(\lambda) \leq T_{+}(\lambda)
$$

where

$$
\begin{aligned}
T_{+}(\lambda)=E & \left(\sum_{j=1}^{\infty} \Gamma_{j}^{-2 / \alpha}\right)^{-1 / 2} \exp \left(-\frac{\lambda^{2}}{2 \beta^{2 / \alpha} \sum_{j=1}^{\infty} \Gamma_{j}^{-2 / \alpha}}\right) \\
& \left.\times \mathbf{1}\left(\sum_{j=1}^{\infty} \Gamma_{j}^{-2 / \alpha}>\lambda^{\theta}, \Gamma_{1}^{-2 / \alpha}>(1-\varepsilon) \sum_{j=1}^{\infty} \Gamma_{j}^{-2 / \alpha}\right)\right) .
\end{aligned}
$$

It follows from our derivation of (4.21) and (4.22) that

$$
\begin{aligned}
\lim _{\lambda \rightarrow \infty} & \lambda^{\alpha+1} T_{+}(\lambda) \\
& =\lim _{\lambda \rightarrow \infty} \lambda^{\alpha+1} E\left(\left(\sum_{j=1}^{\infty} \Gamma_{j}^{-2 / \alpha}\right)^{-1 / 2} \exp \left(-\frac{\lambda^{2}}{2 \beta^{2 / \alpha} \sum_{j=1}^{\infty} \Gamma_{j}^{-2 / \alpha}}\right)\right) \\
& =\frac{\alpha}{2} \Gamma\left(\frac{\alpha+1}{2}\right)\left(2 \beta^{2 / \alpha}\right)^{(\alpha+1) / 2}
\end{aligned}
$$


where to compute the limit we have used Lemma 2.2(i) and the fact that the scale parameter $\sigma$ of $\sum_{j=1}^{\infty} \Gamma_{j}^{-2 / \alpha}$ is equal to $C_{\alpha / 2}^{-2 / \alpha}$ (see Theorem 1.4.5 of [19]).

We now conclude by (4.25), (4.27), (4.29) and (4.30)-(4.32) that

$$
\begin{aligned}
-C \varepsilon & -C h(\delta)+(1-\varepsilon) \frac{\alpha}{2} \Gamma\left(\frac{\alpha+1}{2}\right)\left(2 \beta^{2 / \alpha}\right)^{(\alpha+1) / 2} b_{\alpha}^{-1} \beta^{-1 / \alpha} E\left(R_{1} \mathbf{1}\left(R_{1}>\delta\right)\right) \\
& \leq \liminf _{\lambda \rightarrow \infty} \lambda^{\alpha+1} H_{13}(\lambda) \leq \limsup _{\lambda \rightarrow \infty} \lambda^{\alpha+1} H_{13}(\lambda) \\
& \leq C \varepsilon+C h(\delta)+\frac{\alpha}{2} \Gamma\left(\frac{\alpha+1}{2}\right)\left(2 \beta^{2 / \alpha}\right)^{(\alpha+1) / 2} b_{\alpha}^{-1} \beta^{-1 / \alpha} E\left(R_{1} \mathbf{1}\left(R_{1}>\delta\right)\right)
\end{aligned}
$$

for any $0<\varepsilon, \delta<1$. Letting $\varepsilon$ and $\delta$ go to zero, we conclude that

$$
\begin{aligned}
\lim _{\lambda \rightarrow \infty} \lambda^{\alpha+1} H_{13}(\lambda)= & \frac{\alpha}{2} \Gamma\left(\frac{\alpha+1}{2}\right)\left(2 \beta^{2 / \alpha}\right)^{(\alpha+1) / 2} b_{\alpha}^{-1} \beta^{-1 / \alpha} E\left(R_{1}\right)_{+} \\
= & \alpha 2^{\alpha} \pi^{-1 / 2 \alpha}\left(\Gamma\left(\frac{\alpha+1}{2}\right)\right)^{1+1 / \alpha} C_{\alpha}^{-1 / \alpha} \\
& \times \int_{S}\left|g_{2}(x)\right|\left|g_{1}(x)\right|^{\alpha-1} \mathbf{1}\left(g_{1}(x) g_{2}(x)>0\right) m(d x),
\end{aligned}
$$

thus proving (4.13) and so (4.11) as well.

It remains to prove (4.12) for $i=1,3,4$. We start with $i=1$. We now consider separately the three cases, $1<\alpha<2,0<\alpha<1$ and $\alpha=1$.

Assume first that $1<\alpha<2$. Clearly,

$$
E C_{1}(\lambda) \leq \frac{1}{2 \pi} E\left(\frac{\sigma_{2}}{\sigma_{1}} \exp \left(-\frac{\lambda^{2}}{2\left(\sigma_{1}^{2}-\sigma_{12}^{2} / \sigma_{2}^{2}\right)}\right)\right):=\frac{1}{2 \pi} G(\lambda) .
$$

Observe that, changing a variable, we can write

$$
\left(\sigma_{1}^{2}, \sigma_{2}^{2}, \sigma_{12}\right)=_{d}(X, 0,0)+C\left(s_{1}^{2}, s_{2}^{2}, s_{12}\right),
$$

with the terms in the right-hand side of (4.34) being independent, where $C>0$ is a constant, $X$ has an $S_{\alpha / 2}\left(\sigma_{X}, 0,0\right)$ distribution with

and

$$
\sigma_{X}^{\alpha / 2}=E\left(\left|g_{1}(U)\right|^{\alpha} \mathbf{1}\left(g_{2}(U)=0\right)\right)
$$

$$
\begin{aligned}
s_{1}^{2} & =\sum_{j=1}^{\infty} \Gamma_{j}^{-2 / \alpha} V_{j}^{2}, \\
s_{2}^{2} & =\sum_{j=1}^{\infty} \Gamma_{j}^{-2 / \alpha}, \\
s_{12} & =\sum_{j=1}^{\infty} \Gamma_{j}^{-2 / \alpha} V_{j} .
\end{aligned}
$$

Here $V_{j}, j \geq 1$ is an independent of the Poisson arrivals sequence of i.i.d. random variables such that

$$
V_{1}={ }_{d} g_{1}(\tilde{W}) / g_{2}(\tilde{W}),
$$


where $\tilde{W}$ has a distribution which is absolutely continuous with respect to the law of $U_{1}$, with the Radon-Nikodym derivative given by

$$
\frac{d F_{\tilde{W}}}{d F_{U_{1}}}(x)=\tilde{\beta}^{-1}\left|g_{2}(x)\right|^{\alpha}
$$

and $\tilde{\beta}=E\left|g_{2}\left(U_{1}\right)\right|^{\alpha}$.

Therefore,

$$
G(\lambda)=C^{1 / 2} E\left(\frac{s_{2}}{\left(C s_{1}^{2}+X\right)^{1 / 2}} \exp \left(-\frac{\lambda^{2}}{2\left(C s_{1}^{2}+X-C s_{12}^{2} / s_{2}^{2}\right)}\right)\right) .
$$

Observe that for all $a, b>0$

$$
\begin{aligned}
E\left((X+a+b)^{-1 / 2} \exp \left(-\lambda^{2} / 2(X+a)\right)\right) \\
=E\left((X+a+b)^{-1 / 2} \exp \left(-\lambda^{2} / 2(X+a)\right) \mathbf{1}(X \leq a)\right) \\
\quad+E\left((X+a+b)^{-1 / 2} \exp \left(-\lambda^{2} / 2(X+a)\right) \mathbf{1}(X>a)\right) \\
\leq(a+b)^{-1 / 2} \exp \left(-\lambda^{2} / 4 a\right)+E\left(X^{-1 / 2} \exp \left(-\lambda^{2} / 4 X\right)\right) .
\end{aligned}
$$

Therefore, using (4.37) with

$$
\begin{aligned}
& a=C\left(s_{1}^{2}-s_{12}^{2} / s_{2}^{2}\right), \\
& b=C s_{12}^{2} / s_{2}^{2}
\end{aligned}
$$

and Lemma 2.2(i), we conclude that

$$
G(\lambda) \leq C \lambda^{-(\alpha+1)} E s_{2}+C E\left(\frac{s_{2}}{s_{1}} \exp \left(-\frac{\lambda^{2}}{C\left(s_{1}^{2}-s_{12}^{2} / s_{2}^{2}\right)}\right)\right) .
$$

Recall that $C$ stands for a finite positive constant that may change from line to line. Therefore, (4.12) with $i=1$ will follow if we show that

$$
\lim _{\lambda \rightarrow \infty} \lambda^{\alpha} E\left(\frac{s_{2}}{s_{1}} \exp \left(-\frac{\lambda^{2}}{s_{1}^{2}-s_{12}^{2} / s_{2}^{2}}\right)\right)=0 .
$$

Observe that by (2.12)

$$
\begin{aligned}
E\left(\frac{s_{2}}{s_{1}}\right. & \left.\exp \left(-\frac{\lambda^{2}}{\left(s_{1}^{2}-s_{12}^{2} / s_{2}^{2}\right)}\right)\right) \\
& \leq E\left(s_{2} \exp \left(-\frac{\lambda^{2}}{2\left(s_{1}^{2}-s_{12}^{2} / s_{2}^{2}\right)}\right)\left(s_{1}^{-1} \exp \left(-\frac{\lambda^{2}}{2 s_{1}^{2}}\right)\right)\right) \\
& \leq C \lambda^{-1} E\left(s_{2} \exp \left(-\frac{\lambda^{2}}{2 Z}\right)\right),
\end{aligned}
$$


where $Z=s_{1}^{2}-s_{12}^{2} / s_{2}^{2}$. We have

$$
\begin{aligned}
& Z=s_{1}^{2}-\frac{s_{12}^{2}}{s_{2}^{2}}=\sum_{j=1}^{\infty} \Gamma_{j}^{-2 / \alpha} V_{j}^{2}-\frac{\left(\sum_{j=1}^{\infty} \Gamma_{j}^{-2 / \alpha} V_{j}\right)^{2}}{\sum_{j=1}^{\infty} \Gamma_{j}^{-2 / \alpha}} \\
& =\left(\sum_{j=1}^{\infty} \Gamma_{j}^{-2 / \alpha}\right)^{-1}\left[\Gamma_{1}^{-2 / \alpha}\left(\sum_{j=2}^{\infty} \Gamma_{j}^{-2 / \alpha} V_{j}^{2}+V_{1}^{2} \sum_{j=2}^{\infty} \Gamma_{j}^{-2 / \alpha}\right)\right. \\
& +\sum_{j=2}^{\infty} \Gamma_{j}^{-2 / \alpha} V_{j}^{2} \sum_{j=2}^{\infty} \Gamma_{j}^{-2 / \alpha} \\
& \left.-2 \Gamma_{1}^{-2 / \alpha} V_{1} \sum_{j=2}^{\infty} \Gamma_{j}^{-2 / \alpha} V_{j}-\left(\sum_{j=2}^{\infty} \Gamma_{j}^{-2 / \alpha} V_{j}\right)^{2}\right] \\
& =\left(\sum_{j=1}^{\infty} \Gamma_{j}^{-2 / \alpha}\right)^{-1}\left(\Gamma_{1}^{-2 / \alpha} \sum_{j=2}^{\infty} \Gamma_{j}^{-2 / \alpha}\left(V_{1}-V_{j}\right)^{2}+\sum_{j=2}^{\infty} \Gamma_{j}^{-2 / \alpha} V_{j}^{2} \sum_{j=2}^{\infty} \Gamma_{j}^{-2 / \alpha}\right. \\
& \left.-\left(\sum_{j=2}^{\infty} \Gamma_{j}^{-2 / \alpha} V_{j}\right)^{2}\right) \\
& \leq \sum_{j=2}^{\infty} \Gamma_{j}^{-2 / \alpha}\left(V_{1}-V_{j}\right)^{2}+\sum_{j=2}^{\infty} \Gamma_{j}^{-2 / \alpha} V_{j}^{2}:=Z_{0} .
\end{aligned}
$$

In particular,

$$
E\left(\frac{s_{2}}{s_{1}} \exp \left(-\frac{\lambda^{2}}{\left(s_{1}^{2}-s_{12}^{2} / s_{2}^{2}\right)}\right)\right) \leq C \lambda^{-1} E\left(s_{2} \exp \left(-\frac{\lambda^{2}}{2 Z_{0}}\right)\right)
$$

Note that

$$
\begin{aligned}
P\left(Z_{0}>\lambda\right) & \leq P\left(V_{1}^{2} \sum_{j=2}^{\infty} \Gamma_{j}^{-2 / \alpha}>\lambda / 4\right)+P\left(\sum_{j=2}^{\infty} \Gamma_{j}^{-2 / \alpha} V_{j}^{2}>\lambda / 6\right) \\
& =o\left(\lambda^{-\alpha / 2}\right)
\end{aligned}
$$

by Lemma 2.1(i). Since the conditional distribution of $\Gamma_{1}$ given $\mathscr{F}_{\geq 2}=\sigma\left(\Gamma_{n}\right.$, $n \geq 2)$ is uniform in the interval $\left(0, \Gamma_{2}\right)$, we conclude that

$$
\begin{aligned}
E\left(s_{2} \mid \mathscr{F}_{\geq 2}\right) & \leq E\left(\Gamma_{1}^{-1 / \alpha} \mid \mathscr{F}_{\geq 2}\right)+\left(\sum_{j=2}^{\infty} \Gamma_{j}^{-2 / \alpha}\right)^{1 / 2} \\
& =C \Gamma_{2}^{-1 / \alpha}+\left(\sum_{j=2}^{\infty} \Gamma_{j}^{-2 / \alpha}\right)^{1 / 2}:=\Delta
\end{aligned}
$$


and by Lemma 2.1(i), $P(\Delta>\lambda)=o\left(\lambda^{-\alpha}\right)$ as $\lambda \rightarrow \infty$. Therefore,

$$
\begin{aligned}
E\left(s_{2} \exp \left(-\frac{\lambda^{2}}{2 Z_{0}}\right)\right) & \leq E\left(\Delta \exp \left(-\frac{\lambda^{2}}{2 Z_{0}}\right)\right) \\
& \leq E\left(\left(\Delta^{2}+Z_{0}\right)^{1 / 2} \exp \left(-\frac{\lambda^{2}}{2\left(\Delta^{2}+Z_{0}\right)}\right)\right)=o\left(\lambda^{1-\alpha}\right)
\end{aligned}
$$

by Lemma 2.2(ii). This proves (4.12) with $i=1$ in the case $1<\alpha<2$.

We now turn to the proof of (4.12) for $i=1$ in the case $0<\alpha<1$. Inspecting the above proof for $1<\alpha<2$ shows that the only problem in the present case is that $E \sigma_{2}=C E s_{2}=\infty$. Therefore, we start with the following decomposition. For an $M>0$ write

$$
\begin{aligned}
E C_{1}(\lambda)= & \frac{1}{2 \pi} E\left(\frac{\sigma_{2}}{\sigma_{1}} \mathbf{1}\left(\sigma_{2}>M\right) \sqrt{1-\rho^{2}} \exp \left(-\frac{\lambda^{2}}{2 \sigma_{1}^{2}\left(1-\rho^{2}\right)}\right)\right) \\
& +\frac{1}{2 \pi} E\left(\frac{\sigma_{2}}{\sigma_{1}} \mathbf{1}\left(\sigma_{2} \leq M\right) \sqrt{1-\rho^{2}} \exp \left(-\frac{\lambda^{2}}{2 \sigma_{1}^{2}\left(1-\rho^{2}\right)}\right)\right) \\
:= & G_{1}(\lambda)+G_{2}(\lambda) .
\end{aligned}
$$

The above remark shows that for any $M>0$,

$$
\lim _{\lambda \rightarrow \infty} \lambda^{\alpha} G_{2}(\lambda)=0 .
$$

Therefore, (4.12) with $i=1$ will follow in this case once we establish that for any $\varepsilon>0$ there is an $M>0$ so big that

$$
\limsup _{\lambda \rightarrow \infty} \lambda^{\alpha} G_{1}(\lambda) \leq \varepsilon .
$$

We use (2.12) to conclude that

$$
\begin{aligned}
G_{1}(\lambda)=C E\left(\frac{\sigma_{2}}{\sigma_{1}^{1-\alpha}\left(\sigma_{1}^{\alpha}\left(1-\rho^{2}\right)^{\alpha / 2}\right)} \mathbf{1}\left(\sigma_{2}>M\right)\left(1-\rho^{2}\right)^{(1+\alpha) / 2}\right. \\
\left.\quad \times \exp \left(-\frac{\lambda^{2}}{2 \sigma_{1}^{2}\left(1-\rho^{2}\right)}\right)\right) \\
\leq C \lambda^{-\alpha} E\left(\frac{\sigma_{2}}{\sigma_{1}^{1-\alpha}} \mathbf{1}\left(\sigma_{2}>M\right)\left(1-\rho^{2}\right)^{(1+\alpha) / 2}\right) .
\end{aligned}
$$

Therefore,

$$
\limsup _{\lambda \rightarrow \infty} \lambda^{\alpha} G_{1}(\lambda) \leq C E\left(\frac{\sigma_{2}}{\sigma_{1}^{1-\alpha}} \mathbf{1}\left(\sigma_{2}>M\right)\left(1-\rho^{2}\right)^{(1+\alpha) / 2}\right),
$$

and so (4.45) will follow once we prove that

$$
E\left(\frac{\sigma_{2}}{\sigma_{1}^{1-\alpha}}\left(1-\rho^{2}\right)^{(1+\alpha) / 2}\right)<\infty .
$$


To this end we note that, in the case $0<\alpha \leq 1$, (4.15) extends to

$$
b_{\alpha}^{-2}\left(\sigma_{1}^{2}, \sigma_{2}^{2}, \sigma_{12}\right)={ }_{d} \beta^{2 / \alpha}\left(\sum_{j=1}^{\infty} \Gamma_{j}^{-2 / \alpha}, \sum_{j=1}^{\infty} \Gamma_{j}^{-2 / \alpha} R_{j}^{2}, \sum_{j=1}^{\infty} \Gamma_{j}^{-2 / \alpha} R_{j}\right) .
$$

(Note that it follows by (4.3) and (4.4) that in this case $\sigma_{2}$ cannot have a component independent of $\sigma_{1}$.) Therefore, we can write by (4.47), for a fixed $K>1$,

$$
\begin{aligned}
E\left(\frac{\sigma_{2}}{\left.\sigma_{1}^{1-\alpha}\left(1-\rho^{2}\right)^{(1+\alpha) / 2}\right) \leq}\right. & E\left(\Gamma_{1}^{-1}\left|R_{1}\right|\left(1-\rho^{2}\right)^{1 / 2}\right)+E\left(\sum_{j=2}^{K} \Gamma_{j}^{-1}\left|R_{j}\right|\right) \\
& +E\left(\frac{\left(\sum_{j=K+1}^{\infty} \Gamma_{j}^{-2 / \alpha} R_{j}^{2}\right)^{1 / 2}}{\left(\sum_{j=1}^{\infty} \Gamma_{j}^{-2 / \alpha}\right)^{(1-\alpha) / 2}}\right) \\
:= & M_{1}+M_{2}+M_{3} .
\end{aligned}
$$

It follows from (4.17) that $M_{2}<\infty$ for every $K \geq 2$. Furthermore,

$$
\begin{aligned}
M_{3} & \leq E\left|R_{1}\right| E\left(\frac{\sum_{j=K+1}^{\infty} \Gamma_{j}^{-1 / \alpha}}{\left(\sum_{j=1}^{\infty} \Gamma_{j}^{-2 / \alpha}\right)^{(1-\alpha) / 2}}\right) \\
& \leq C\left(E\left(\sum_{j=K+1}^{\infty} \Gamma_{j}^{-1 / \alpha}\right)^{2} E\left(\sum_{j=1}^{\infty} \Gamma_{j}^{-2 / \alpha}\right)^{-(1-\alpha)}\right)^{1 / 2}<\infty
\end{aligned}
$$

as long as $K$ is large enough, once again because positive $\alpha$-stable random variables have negative moments of all orders. Therefore, (4.46) will follow if we establish that $M_{1}<\infty$. To do so we recall that by (4.41) we have

$$
1-\rho^{2} \leq \frac{1}{\sigma_{1}^{2} \sigma_{2}^{2}}\left(\Gamma_{1}^{-2 / \alpha} \sum_{j=2}^{\infty} \Gamma_{j}^{-2 / \alpha}\left(R_{1}-R_{j}\right)^{2}+\sum_{j=2}^{\infty} \Gamma_{j}^{-2 / \alpha} R_{j}^{2} \sum_{j=2}^{\infty} \Gamma_{j}^{-2 / \alpha}\right) .
$$

Therefore,

$$
\begin{aligned}
M_{1} \leq & E\left(\frac{\Gamma_{1}^{-(1+1 / \alpha)}\left|R_{1}\right|}{\sigma_{1} \sigma_{2}}\left(\sum_{j=2}^{\infty} \Gamma_{j}^{-2 / \alpha}\left(R_{1}-R_{j}\right)^{2}\right)^{1 / 2}\right) \\
& +E\left(\frac{\Gamma_{1}^{-1}\left|R_{1}\right|}{\sigma_{1} \sigma_{2}}\left(\sum_{j=2}^{\infty} \Gamma_{j}^{-2 / \alpha} R_{j}^{2} \sum_{j=2}^{\infty} \Gamma_{j}^{-2 / \alpha}\right)^{1 / 2}\right):=M_{11}+M_{12} .
\end{aligned}
$$

Notice that

$$
\left(\sum_{j=2}^{\infty} \Gamma_{j}^{-2 / \alpha}\left(R_{1}-R_{j}\right)^{2}\right)^{1 / 2} \leq 2^{1 / 2}\left(\sum_{j=2}^{\infty} \Gamma_{j}^{-2 / \alpha} R_{j}^{2}\right)^{1 / 2}+2^{1 / 2}\left|R_{1}\right|\left(\sum_{j=2}^{\infty} \Gamma_{j}^{-2 / \alpha}\right)^{1 / 2},
$$


and so we can bound $M_{11}$ from above as follows:

$$
\begin{aligned}
M_{11} & \leq C E\left(\Gamma_{1}^{-1}\left|R_{1}\right|\left(\frac{\sum_{j=2}^{\infty} \Gamma_{j}^{-2 / \alpha} R_{j}^{2}}{\sum_{j=1}^{\infty} \Gamma_{j}^{-2 / \alpha} R_{j}^{2}}\right)^{1 / 2}\right)+C E\left(\Gamma_{1}^{-1}\left|R_{1}\right|\left(\frac{\sum_{j=2}^{\infty} \Gamma_{j}^{-2 / \alpha}}{\sum_{j=1}^{\infty} \Gamma_{j}^{-2 / \alpha}}\right)^{1 / 2}\right) \\
& \leq C E\left(\left|R_{1}\right|^{1-\alpha} \frac{\left(\sum_{j=2}^{\infty} \Gamma_{j}^{-2 / \alpha} R_{j}^{2}\right)^{1 / 2}}{\left(\sum_{j=1}^{\infty} \Gamma_{j}^{-2 / \alpha} R_{j}^{2}\right)^{(1-\alpha) / 2}}\right)+C E\left(\frac{\left(\sum_{j=2}^{\infty} \Gamma_{j}^{-2 / \alpha}\right)^{1 / 2}}{\left(\sum_{j=1}^{\infty} \Gamma_{j}^{-2 / \alpha}\right)^{(1-\alpha) / 2}}\right) \\
& \leq C E\left(\left|R_{1}\right|^{1-\alpha}\left(\sum_{j=2}^{\infty} \Gamma_{j}^{-2 / \alpha} R_{j}^{2}\right)^{\alpha / 2}\right)+C E\left(\left(\sum_{j=2}^{\infty} \Gamma_{j}^{-2 / \alpha}\right)^{\alpha / 2}\right)<\infty
\end{aligned}
$$

by (4.17) and Lemma 2.1(i). Furthermore,

$$
M_{12} \leq E\left(\Gamma_{1}^{-1}\left|R_{1}\right|\left(\frac{\sum_{j=2}^{\infty} \Gamma_{j}^{-2 / \alpha}}{\sum_{j=1}^{\infty} \Gamma_{j}^{-2 / \alpha}}\right)^{1 / 2}\right)<\infty
$$

as above. This proves (4.46), and so we have proved (4.12) with $i=1$ in the case $0<\alpha<1$.

This leaves us only with the case $\alpha=1$ to consider. The argument of $0<\alpha<1$ works verbatim in the present case, provided we can show that for a $K$ large enough,

$$
E\left(\left(\sum_{j=K}^{\infty} \Gamma_{j}^{-2} R_{j}^{2}\right)^{1 / 2}\right)<\infty .
$$

However, it follows from (4.4) that

$$
E\left(\left|R_{1}\right| \log \left(1+R_{1}^{2}\right)\right)<\infty,
$$

and so we can use Lemma 2.1(ii) to conclude that for $K \geq 3$,

$$
E\left(\left(\sum_{j=K}^{\infty} \Gamma_{j}^{-2} R_{j}^{2}\right)^{1 / 2}\right) \leq C E\left(\left(\sum_{j=K}^{\infty} j^{-2} R_{j}^{2}\right)^{1 / 2}\right)<\infty .
$$

This proves (4.51), and so (4.12) with $i=1$ has been proved in all cases.

We now turn to proving (4.12) for $i=3$ and $i=4$. Using the formula

$$
\int_{t}^{\infty} \exp \left(-y^{2} / 2\right) d y \leq t^{-1} \exp \left(-t^{2} / 2\right), \quad t>0
$$

we immediately conclude that

$$
\begin{aligned}
E C_{3}(\lambda) & =E\left(\frac{\sigma_{2} \rho}{2 \pi \sigma_{1}^{2}} \lambda \exp \left(-\frac{\lambda^{2}}{2 \sigma_{1}^{2}}\right) \int_{-\lambda \rho /\left(\sigma_{1} \sqrt{1-\rho^{2}}\right)}^{\infty} \exp \left(-\frac{y^{2}}{2}\right) d y \mathbf{1}(\rho \leq 0)\right) \\
& \leq E\left(\frac{\sigma_{2}}{\sigma_{1}} \frac{\sqrt{1-\rho^{2}}}{2 \pi} \exp \left(-\frac{\lambda^{2}}{2 \sigma_{1}^{2}\left(1-\rho^{2}\right)}\right) \mathbf{1}(\rho \leq 0)\right) \leq E C_{1}(\lambda),
\end{aligned}
$$


and so (4.12) for $i=3$ follows from the already proven case $i=1$. In exactly the same manner we see that

$$
\begin{aligned}
E\left|C_{4}(\lambda)\right| & =E\left(\frac{\sigma_{2} \rho}{2 \pi \sigma_{1}^{2}} \lambda \exp \left(-\frac{\lambda^{2}}{2 \sigma_{1}^{2}}\right) \int_{-\infty}^{-\lambda \rho /\left(\sigma_{1} \sqrt{1-\rho^{2}}\right)} \exp \left(-\frac{y^{2}}{2}\right) d y \mathbf{1}(\rho>0)\right) \\
& \leq E\left(\frac{\sigma_{2}}{\sigma_{1}} \frac{\sqrt{1-\rho^{2}}}{2 \pi} \exp \left(-\frac{\lambda^{2}}{2 \sigma_{1}^{2}\left(1-\rho^{2}\right)}\right) \mathbf{1}(\rho>0)\right) \leq E C_{1}(\lambda),
\end{aligned}
$$

and so (4.12) for $i=4$ also follows from the case $i=1$.

This completes the proof of the proposition in the case when the control measure $m$ of the $S \alpha S$ random measure $M$ is a probability measure. In the general case of a $\sigma$-finite control measure $m$, we proceed as in the proof of Theorem 1.1. That is, let $\eta$ be a probability measure on $S$ equivalent to $m$. Let $b(x)=(d m / d \eta)(x), x \in S$. Then we can represent (in distribution) the random vector $(U, W)$ in the form

$$
\begin{gathered}
U=\int_{S} g_{1}(x) b(x)^{1 / \alpha} M_{1}(d x), \\
W=\int_{S} g_{2}(x) b(x)^{1 / \alpha} M_{1}(d x),
\end{gathered}
$$

where $M_{1}$ is now an $S \alpha S$ random measure on $S$ with a control measure $\eta$. Observe that we have reduced the situation to that of the control measure being a probability measure, with the new functions

$$
\tilde{g}_{1}(x)=g_{1}(x) b(x)^{1 / \alpha}
$$

and

$$
\tilde{g}_{2}(x)=g_{2}(x) b(x)^{1 / \alpha} .
$$

It is obvious that the functions $\tilde{g}_{1}$ and $\tilde{g}_{2}$ satisfy (4.3) or (4.4) (with $m$ replaced by $\eta$ ) whenever $g_{1}$ and $g_{2}$ do. Therefore, we have (4.5) with

$$
\begin{aligned}
\kappa_{\alpha}\left(g_{1}, g_{2}\right)= & \int_{S}\left|\tilde{g}_{2}(x)\right|\left|\tilde{g}_{1}(x)\right|^{\alpha-1} \mathbf{1}\left(\tilde{g}_{1}(x) \tilde{g}_{2}(x)>0\right) \eta(d x) \\
& \times \int_{S}\left|g_{2}(x)\right|\left|g_{1}(x)\right|^{\alpha-1} \mathbf{1}\left(g_{1}(x) g_{2}(x)>0\right) m(d x),
\end{aligned}
$$

and so the proposition has been proved in its full generality.

REMARK. As mentioned above, Proposition 4.1 is of interest independent of its use in the proof of Theorem 1.1. It is known (see [8]) that the conditional law of $W / \lambda$ given $U=\lambda$ converges, as $\lambda \rightarrow \infty$, to the normalized spectral 
measure (mapped to the real line) of the $S \alpha S$ random vector $(U, W)$ (thus showing that the set of conditional distributions of bivariate stable random vectors is weakly dense in the set of all univariate probability distributions - very much unlike the normal case). Proposition 4.1 gives, in the symmetric case, convergence of certain moments of these conditional distributions. Indeed, it shows that the expectation of the positive part of $W / \lambda$ given $U=\lambda$ [which is finite even in the case $0<\alpha \leq 1$ under the assumptions (4.3) and (4.4)] converges to the expectation of the positive part of a random variable distributed according to the normalized spectral measure, mapped to the real line.

Moreover, an argument identical to that of Proposition 4.1 shows that both the expectation of the whole of $W / \lambda$, given $U=\lambda$, and the expectation of the absolute value of $W / \lambda$, given $U=\lambda$, which are finite under the same assumptions, converge, correspondingly, to the expectation and the expectation of the absolute value, of the above random variable distributed according to the normalized spectral measure, mapped to the real line.

Acknowledgment. The authors would like to thank Patrik Albin for drawing their attention to Marcus's paper [11].

\section{REFERENCES}

[1] Adler, R., Samorodnitsky, G. and Gadrich, T. (1993). The expected number of level crossings for stationary, harmonizable, symmetric, stable processes. Ann. Appl. Probab. 3 553-575.

[2] BRILlinger, D. R. (1972). On the number of solutions of systems of random equations. Ann. Math. Statist. 43 534-540.

[3] Cambanis, S., Rosiński, J. and Woyczynski, W. A. (1985). Convergence of quadratic forms in $p$-stable random variables and $\theta_{p}$-radonifying operators. Ann. Probab. 13 885-897.

[4] Cioczen-Georges, R. and TAqQu, M. S. (1994). How do conditional moments of stable vectors depend on the spectral measure? Stochastic Process. Appl. 54 95-112.

[5] Cioczek-Georges, R. and Taqqu, M. S. (1995). Necessary conditions for the existence of conditional moments of stable random variables. Stochastic Process. Appl. 56 233-246.

[6] Cramér, H. and Leadbetter, M. R. (1967). Stationary and Related Stochastic Processes. Wiley, New York.

[7] Geman, D. and Horowitz, J. (1973). Occupation times for smooth stationary processes. Ann. Probab. 1 131-137.

[8] Hauser, R. and Matzinger, H. (1995). Conditional distributions of stable vectors. Unpublished manuscript.

[9] Leadbetter, M. R., Lindgren, G. and Rootzén, H. (1983). Extremes and Related Properties of Random Sequences and Processes. Springer, New York.

[10] Ledoux, M. and Talagrand, M. (1991). Probability in Banach Spaces: Isoperimetry and Processes. Springer, New York.

[11] Marcus, M. B. (1977). Level crossings of a stochastic process with absolutely continuous sample paths. Ann. Probab. 5 52-71.

[12] Marcus, M. B. (1989). Some bounds for the expected number of level crossings of symmetric harmonizable $p$-stable processes. Stochastic Process. Appl. 33 217-231.

[13] Maruyama, G. (1970). Infinitely divisible processes. Theory Probab. Appl. 15 1-22.

[14] MichnA, Z. and Rychlik, I. (1992). Characteristics of upcrossings for stable processes. Technical report, Dept. Mathematical Statistics, Lund Institute of Technology. 
[15] MichnA, Z. and RychliK, I. (1992). Expected number of level crossings for certain symmetric $\alpha$-stable processes. Comm. Statist. - Stochastic Models 11 1-19.

[16] Rice, S. O. (1945). Mathematical analysis of random noise. Bell System Tech. J. 24 46-156.

[17] Rosiński, J. (1986). On stochastic integral representation of stable processes with sample paths in Banach spaces. J. Multivariate Anal. 20 277-302.

[18] Rosí́ski, J. (1995). On the structure of stationary stable processes. Ann. Probab. 23 1163-1187.

[19] Samorodnitsky, G. and Taqqu, M. S. (1994). Stable Non-Gaussian Random Processes. Chapman and Hall, New York.

FACULTY OF INDUSTRIAL ENGINEERING

AND MANAGEMENT

TEChNiON-IsRaEl Institute of TeChNOLOGY

HAIFA, 32000

ISRAEL

AND

DEPARTMENT OF Statistics

University of NoRTH CAROLINA

Chapel Hill, North Carolina 27599-3260

E-MAIL: adler@stat.unc.edu
School of Operations Research AND INDUSTRIAL ENGINEERING

CORNELL UNIVERSITY

ITHACA, NEW YoRK 14853

E-MAIL: gennady@orie.cornell.edu 